\title{
Constraints on Lorentz violation from clock-comparison experiments
}

\author{
V. Alan Kostelecký and Charles D. Lane \\ Physics Department, Indiana University, Bloomington, Indiana 47405
}

(Received 29 April 1999; published 12 November 1999)

\begin{abstract}
Constraints from clock-comparison experiments on violations of Lorentz and $C P T$ symmetry are investigated in the context of a general Lorentz-violating extension of the standard model. The experimental signals are shown to depend on the atomic and ionic species used as clocks. Certain experiments usually regarded as establishing comparable bounds are in this context sensitive to different types of Lorentz violation. Some considerations relevant to possible future measurements are presented. All these experiments are potentially sensitive to Lorentz-violating physics at the Planck scale. [S0556-2821(99)01223-0]
\end{abstract}

PACS number(s): 11.30.Er, 12.20.Fv, 12.60.-i, 41.20.Jb

\section{INTRODUCTION}

Covariance under Lorentz transformations is a feature of modern descriptions of nature at the fundamental level. These transformations include both spatial rotations and boosts, linked through the relativistic connection between space and time. Experimental investigations of rotation symmetry therefore play a crucial role in testing the framework of theories such as the $\mathrm{SU}(3) \times \mathrm{SU}(2) \times \mathrm{U}(1)$ standard model of particle physics.

Clock-comparison experiments [1-7] form a class of particularly sensitive tests of rotation invariance and hence of Lorentz symmetry. The basic idea is to constrain possible spatial anisotropies by bounding the variation in frequency of a given clock as its orientation changes. In practice, the most precise limits are obtained by comparing the frequencies of two different clocks as they rotate with the Earth. The clocks used are typically atoms or ions, and the relevant frequencies are usually those of the light emitted or absorbed in hyperfine or Zeeman transitions. Experiments of this type face a number of important challenges, in particular the elimination of systematic effects from mundane causes. Nonetheless, remarkable sensitivity to possible Lorentz violations can be attained.

In the present work, a theoretical interpretation of clockcomparison experiments is performed in the context of a general extension of the standard model of particle physics incorporating a consistent microscopic theory of Lorentz violation, including terms both even and odd under CPT [8]. This standard-model extension must emerge from any underlying theory that generates the standard model and contains spontaneous Lorentz violation [9]. It maintains both the usual gauge structure based on $\mathrm{SU}(3) \times \mathrm{SU}(2) \times \mathrm{U}(1)$ symmetry and the usual power-counting renormalizability. It also has a variety of other desirable features, including energymomentum conservation, observer Lorentz covariance, conventional quantization, and hermiticity, while microcausality and positivity of the energy are expected.

From the perspective of the present work, this standardmodel extension is advantageous not only because it provides a consistent and general theoretical framework for studying Lorentz violations but more specifically because it is quantitative and at the level of the known elementary particles. The Lagrangian of the theory is formed using fields for the elementary particles, and the possible Lorentz viola- tions for each type of particle and interaction are controlled by parameters whose values are to be determined by experiment. Since atoms and ions are composed of these elementary particles, the behavior of different atoms and ions under rotations and boosts is determined by the parameters for Lorentz violation in the theory. It is therefore possible within this framework to provide a quantitative comparative analysis of clock-comparison experiments performed with different substances and to examine interesting possibilities for future experiments. Both of these are undertaken in the present work.

Although many tests of Lorentz and $C P T$ symmetry exist [10-12], the clock-comparison ones considered here are among the relatively few experiments that could be sensitive to the minuscule effects motivating the standard-model extension. For sensitive experiments of any type, the standardmodel extension provides a quantitative and coherent framework at the level of the standard model and quantum electrodynamics (QED) within which to analyze and compare the results obtained and, in favorable circumstances, to predict possible observable signals. Prior to this work, the standard-model extension has been used to examine possible bounds on Lorentz and CPT violation from measurements of neutral-meson oscillations [13-16], from tests of QED in Penning traps [17-21], from photon birefringence [22,8,23], from hydrogen and antihydrogen spectroscopy [24,25], and from baryogenesis [26].

The structural outline of the paper is as follows. Section II presents our theoretical procedures and discusses associated issues. Following some general remarks, Sec. II A is devoted to the relativistic Lagrangian and nonrelativistic Hamiltonian used for our analysis. The expressions for the Lorentzviolating shifts in atomic and ionic energy levels are obtained in Sec. II B. Some comments on procedures to evaluate the resulting expectation values are provided in Sec. II C. The incorporation of geometrical effects due to the Earth's rotation and the derivation of theoretically observable signals is given in Sec. IID. Section III applies this analysis, both to published experiments and to future possibilities. Some comments about derivations relevant to specific experiments are relegated to the Appendix.

\section{THEORY}

Clock-comparison experiments involve measurements of transitions between energy levels in atoms or ions. Examin- 
ing shifts in these levels is therefore of central interest in a theoretical analysis of possible effects arising from Lorentz violation. Most atoms and ions are comprised of many elementary particles interacting together to form a system of considerable complexity, so a complete $a b$ initio calculation of energy-level shifts from the various sources of Lorentz violation is impractical. However, any effects from possible Lorentz violation must be minuscule, so theoretical calculations can proceed perturbatively and it suffices to determine only the leading-order effects on the atomic or ionic energy levels.

The Lorentz violations in the standard-model extension can be viewed as arising from the interaction of elementary particles with background expectation values of Lorentz tensor fields in the vacuum, somewhat like the effect of the electromagnetic field of a crystal on the behavior of a charged particle passing through it [8]. There are Lorentzviolating effects both in the quadratic terms in the lagrangian and in the interactions. The Lorentz violations in the quadratic terms induce modifications to the usual free-particle propagators, producing shifts in the conventional freeparticle energies that vary with physical properties of the particle such as the spin and boost magnitudes and orientations. The Lorentz violations in the interactions induce modifications to the vertices describing the particle interactions, and they therefore necessarily involve the associated interaction coupling constant.

In the present work, we proceed under the usual perturbative assumption that effects associated with free propagation are larger than those associated with interactions and that the latter can therefore be disregarded in extracting the leadingorder signals. This approximation is likely to be good when the elementary particles are electrons, but may be questionable for nuclear calculations with protons or neutrons where the strong interaction is involved. Given this assumption, the dominant contribution to the perturbative Lorentz-violating energy-level shifts in an atom or ion can be obtained by summing over individual energy shifts experienced by the component particles as if they were freely propagating in the background expectation values. The energy shifts contributed by each individual particle can be found by taking expectation values of the (nonrelativistic) perturbative Hamiltonian describing the Lorentz violation in the multiparticle unperturbed atomic or ionic state.

Rough dimensional estimates can be used to gain some insight about the relative importance of the perturbative approximations made. On dimensional grounds, the energy shift of the levels of an atom or ion must have the form of a product of some parameter for Lorentz violation with a function that is independent of all such parameters. This function can be taken to be dimensionless (in natural units, $\hbar=c=1$ ) by absorbing a suitable power of a particle mass in the parameter for Lorentz violation as needed. The function can thus be approximated by a multivariable Taylor expansion in dimensionless combinations of physical quantities: expectation values of various angular momentum operators, relativistic correction factors involving the squared ratio of momentum to mass, and interaction energies per mass. The expectation values of angular momenta are of order unity.
The relativistic correction factors are of order $10^{-2}$ for nucleons and $10^{-5}$ for electrons. The electromagneticinteraction energies per mass are of the order of $10^{-5}$ for electrons in atoms and $10^{-3}$ for protons in a nucleus, while the strong-interaction energies per mass are of order $10^{-2}$. In principle, there is an additional dimensionless combination involving the ratio of the energy of the external electromagnetic field to the mass, but even in magnetic fields of order 1 $\mathrm{T}$ this is only of order $10^{-10}$ for electrons and $10^{-16}$ for protons. These crude estimates suggest that the largest Lorentz-violation effects come from expectation values of angular momenta and spins. This is confirmed by the explicit calculations that follow.

The exceptional sensitivity of clock-comparison experiments suggests that useful bounds might in principle also be obtained from subleading Lorentz-violating effects, particularly if different parameters for Lorentz violation appear. However, the exact calculation of subleading effects is challenging. They arise both from relativistic corrections to the free propagation and from corrections coupling the Lorentz violations to the interactions. The dominant role of the strong force at the nuclear level makes the latter corrections difficult to determine reliably. We therefore restrict attention in the present work to relativistic corrections arising from the free propagation of the component particles in the background expectation values. These corrections can be calculated in perturbation theory from subleading terms in the nonrelativistic Hamiltonian. They provide a reasonable sense of the kinds of bound implied by subleading effects on clockcomparison experiments.

The remainder of this section provides the theoretical basis for our results. Section II A presents the general quadratic relativistic Lagrangian for a spin $-\frac{1}{2}$ fermion, allowing for the possibility of Lorentz violation. It is a suitable limit of the standard-model extension and can be used to describe the free propagation of the individual electrons, protons, and neutrons forming the atom or ion of interest. The associated nonrelativistic Hamiltonian is also presented. Section II B derives expressions for the energy-level shifts of a general atom or ion by taking suitable expectation values of the perturbative Lorentz-violating terms in the nonrelativistic Hamiltonian. A few more technical issues associated with evaluation of matrix elements in light of the many-body nuclear and electronic physics are considered in Sec. II C. The connection to experimental observables is treated in Sec. II D, which examines the effect of geometrical factors and the Earth's rotation on the energy-level shifts in various clock-comparison experiments.

\section{A. Lagrangian and Hamiltonian}

A general expression for the quadratic Hermitian Lagrangian describing a single spin- $\frac{1}{2}$ Dirac fermion $\psi$ of mass $m$ in the presence of Lorentz violation is [8]

$$
\mathcal{L}=\frac{1}{2} i \bar{\psi} \Gamma_{\nu} \stackrel{\leftrightarrow}{\partial}^{\nu} \psi-\bar{\psi} M \psi
$$

where 


$$
M:=m+a_{\mu} \gamma^{\mu}+b_{\mu} \gamma_{5} \gamma^{\mu}+\frac{1}{2} H_{\mu \nu} \sigma^{\mu \nu}
$$

and

$\Gamma_{\nu}:=\gamma_{\nu}+c_{\mu \nu} \gamma^{\mu}+d_{\mu \nu} \gamma_{5} \gamma^{\mu}+e_{\nu}+i f_{\nu} \gamma_{5}+\frac{1}{2} g_{\lambda \mu \nu} \sigma^{\lambda \mu}$

This represents an extension of the usual Lagrangian for a massive Dirac fermion field. The Dirac matrices $\left\{1, \gamma_{5}, \gamma^{\mu}, \gamma_{5} \gamma^{\mu}, \sigma^{\mu \nu}\right\}$ appearing in Eqs. (2) and (3) all have conventional properties.

The Lorentz violation in Eq. (1) is governed by the parameters $a_{\mu}, b_{\mu}, c_{\mu \nu}, d_{\mu \nu}, e_{\mu}, f_{\mu}, g_{\lambda \mu \nu}$, and $H_{\mu \nu}$, which could arise as expectation values of Lorentz tensors following spontaneous Lorentz breaking in an underlying theory. The Hermiticity of $\mathcal{L}$ means that all the parameters are real. The parameters appearing in $M$ have dimensions of mass, while those in $\Gamma$ are dimensionless. Both $c_{\mu \nu}$ and $d_{\mu \nu}$ are traceless, while $H_{\mu \nu}$ is antisymmetric and $g_{\lambda \mu \nu}$ is antisymmetric in its first two indices. The parameters $e_{\mu}, f_{\mu}$, and $g_{\lambda \mu \nu}$ are incorporated here for generality. Gauge invariance and renormalizability exclude these in the standard-model extension, so if $\psi$ represents an electron field they are absent or suppressed relative to the others. However, the situation is less clear if $\psi$ represents a proton or neutron because these particles are composites of valence quarks in a sea of other particles. The strong binding involved might generate effective terms governed by appreciable parameters $e_{\mu}, f_{\mu}, g_{\lambda \mu \nu}$ despite their absence in the standard-model extension itself.

The field operators in the terms with coefficients $a_{\mu}, b_{\mu}$, $e_{\mu}, f_{\mu}$, and $g_{\lambda \mu \nu}$ are odd under $C P T$, while the others are even. Since both the particle field and the background tensor expectation values transform covariantly under rotations or boosts of an observer's inertial frame, the Lagrangian (1) remains invariant under observer Lorentz transformations. However, the background expectation values are unaffected by direct rotations or boosts of the particle or localized field in the same observer inertial frame, so the Lagrangian transforms nontrivially under particle Lorentz transformations [8].

All these parameters are expected to be minuscule, in which case the Lorentz-breaking effects are likely to be detectable only in experiments of exceptional sensitivity. Credible estimates for the order of magnitude of the parameters are difficult to make in the absence of a realistic underlying theory. Various sources of suppression might arise. For example, if the origin of the Lorentz violation lies at the Planck scale $M_{P}$, one natural suppression factor would be some power of the ratio $r \approx m_{l} / M_{P}$, where $m_{l}$ is a low-energy scale. Another natural factor could emerge from the coupling strengths in the underlying theory and could produce suppressions similar to those for the particle masses in the usual standard model, arising from the Yukawa couplings to the Higgs scalar field. Other substantial suppression factors might also appear. A further potential complication is that some parameters might be much more heavily suppressed than others. In what follows, we make no specific assumptions about the absolute or relative magnitudes of the parameters for Lorentz violation other than to suppose they are minuscule.

To determine the leading-order effects of the Lorentz violation, it suffices to use a nonrelativistic description for the particles comprising the electron cloud and the nuclear core of the atoms or ions involved in the clock-comparison experiment. We therefore need the nonrelativistic Hamiltonian $h$ associated with the Lagrangian (1). The relativistic Hamiltonian can be found from the Lagrangian $\mathcal{L}$ and the nonrelativistic momentum-space Hamiltonian $h$ can then be derived [27] using Foldy-Wouthuysen techniques [28]. The quantity of interest is the perturbation Hamiltonian $\delta h$ for Lorentz violation, which is the difference $\delta h \equiv h-\hat{h}$ between $h$ and the usual free-particle Foldy-Wouthuysen Hamiltonian $\hat{h}$.

Including all types of operator that arise from Eq. (1) and keeping terms to second order in the Foldy-Wouthuysen expansion for the nonrelativistic Hamiltonian, we find

$$
\begin{aligned}
\delta h= & \left(a_{0}-m c_{00}-m e_{0}\right)+\left(-b_{j}+m d_{j 0}-\frac{1}{2} m \varepsilon_{j k l} g_{k l 0}+\frac{1}{2} \varepsilon_{j k l} H_{k l}\right) \sigma^{j}+\left[-a_{j}+m\left(c_{0 j}+c_{j 0}\right)+m e_{j}\right] \frac{p_{j}}{m} \\
& +\left[b_{0} \delta_{j k}-m\left(d_{k j}+d_{00} \delta_{j k}\right)-m \varepsilon_{k l m}\left(\frac{1}{2} g_{m l j}+g_{m 00} \delta_{j l}\right)-\varepsilon_{j k l} H_{l 0}\right] \frac{p_{j}}{m} \sigma^{k}+\left[m\left(-c_{j k}-\frac{1}{2} c_{00} \delta_{j k}\right)\right] \frac{p_{j} p_{k}}{m^{2}} \\
& +\left\{\left[m\left(d_{0 j}+d_{j 0}\right)-\frac{1}{2}\left(b_{j}+m d_{j 0}+\frac{1}{2} m \varepsilon_{j m n} g_{m n 0}+\frac{1}{2} \varepsilon_{j m n} H_{m n}\right)\right] \delta_{k l}+\frac{1}{2}\left(b_{l}+\frac{1}{2} m \varepsilon_{l m n} g_{m n 0}\right) \delta_{j k}\right. \\
& \left.-m \varepsilon_{j l m}\left(g_{m 0 k}+g_{m k 0}\right)\right\} \frac{p_{j} p_{k}}{m^{2}} \sigma^{l} .
\end{aligned}
$$

Here, Lorentz indices are split into timelike and spacelike cartesian components: $\mu \equiv 0$ and $j=1,2,3$. Repeated indices are understood to be summed. The totally antisymmetric rotation tensor $\varepsilon_{j k l}$ satisfies $\varepsilon_{123}=+1$, with $\varepsilon^{j k l}=-\varepsilon_{j k l}$ as a consequence of the embedding of the rotation group in the
Lorentz group and the usual adoption of a metric tensor of signature -2 . The three-momentum of the particle is denoted by $p_{j}$, while the Pauli matrices are denoted by $\sigma^{j}$ and obey $\left[\sigma^{j}, \sigma^{k}\right]=2 i \varepsilon_{j k l} \sigma^{l}$ as usual.

The leading-order terms in Eq. (4) are those unsuppressed 
by powers of $p_{j} / m$. Nonrelativistic experiments with ordinary matter are therefore dominantly sensitive to the particular combinations of parameters for Lorentz violation appearing in these terms. A further separation of these combinations at the leading-order nonrelativistic level would require experiments with antimatter. However, the exceptional sensitivity of clock-comparison experiments means that they could in principle place interesting (but weaker) bounds on further combinations of parameters for Lorentz violation arising in subleading terms of the nonrelativistic Hamiltonian. Disregarding interactions, the relevant effects arise from terms in $\delta h$ of second order in $p_{j} / m$. In what follows, we investigate bounds arising from all the terms displayed in Eq. (4).

The nonrelativistic Hamiltonian (4) describes speciesspecific energy shifts depending on the spin and momentum of individual particles. Certain other approaches to Lorentz violation also suggest effects of this type. Some indication of their relation to the present work can be obtained by comparing them to the Hamiltonian (4) and its associated underlying theory. A complete review lies beyond the scope of this paper, and we limit ourselves here to only a few remarks $[11,12]$.

Among the purely phenomenological treatments that have been widely applied to clock-comparison experiments is the TH $\epsilon \mu$ formalism [29]. This provides a parametrization of the dynamics of classical charged pointlike test particles in an external spherically symmetric and static gravitational field. It has been used to probe quantitatively the foundations of theories of gravity, including the possibility of deviations from local Lorentz invariance. The TH $\epsilon \mu$ formalism differs qualitatively in several respects from the standard-model extension studied here. Thus, the latter has observer Lorentz covariance and provides an apparently consistent theory at the quantum level for all nongravitational forces, but in its present formulation does not explicitly include gravity (although gravity is implicitly present and some aspects of its inclusion have been investigated [9]). Neglecting gravity, the overlap between the theories is perhaps greatest in the purely electromagnetic sector, where the $T H \epsilon \mu$ parameters $\mu$ and $\epsilon$ have similar features to certain components of the parameter $\left(k_{F}\right)_{\kappa \lambda \mu \nu}$ in the standard-model extension [8].

Several of the existing clock-comparison experiments have been analyzed using models with a term of the form $K \hat{p} \cdot \vec{\sigma}$ in the Hamiltonian, where $\hat{p}$ is a unit vector in the direction of the particle momentum with respect to some preferred frame. A term of this type has been considered by Nielsen and Picek [30], for example, who regard the observed Lorentz symmetry in nature as a low-energy manifestation in a fundamental theory without Lorentz invariance. Among the terms in the Hamiltonian (4) are ones proportional to $\delta_{j k} p_{j} \sigma^{k}$. Since the standard-model extension is covariant under observer Lorentz transformations, its nonrelativistic Hamiltonian has identical form to lowest nonrelativistic order in all inertial frames, and so the coefficients of these terms can emulate $K$. Note, however, that the observer Lorentz covariance also ensures that the standardmodel extension strictly has no preferred frame. There may be a frame in which certain parameters take a simple form [for example, if $b_{\mu}$ is timelike then $b_{\mu} \equiv(b, \overrightarrow{0})$ in some frame], but there is no reason a priori to suppose that this frame is the same for all parameters in the standard-model extension.

A phenomenological approach to Lorentz violation at high energies has recently been presented by Coleman and Glashow [31]. It assumes the existence of a preferred frame in which there are small Lorentz-violating couplings that are $\mathrm{CPT}$ and rotation invariant. The preferred frame is identified with that of the cosmic microwave background, and attention is restricted to renormalizable Lorentz-violating operators that dominate at high energies. These operators are in correspondence with a subset of those appearing in the standardmodel extension. For example, with the Coleman-Glashow assumptions the Lagrangian (1) reduces in the preferred frame to one in which effectively only the parameters $c_{00}$ and $d_{00}$ are nonzero.

\section{B. Atomic and ionic energy-level shifts}

In this subsection, we apply the nonrelativistic Hamiltonian presented in Sec. II A to obtain perturbative shifts of atomic or ionic energy levels arising from Lorentz violation. Let the atom or ion $W$ under consideration have $N_{w}$ particles of type $w$, where $w$ is $p$ for the proton, $n$ for the neutron, and $e$ for the electron. The multiparticle Hamiltonian describing $W$ has one (rotationally invariant) component arising from conventional physics and a second (perturbative, Lorentzviolating) component $h^{\prime}$ that is linear in the parameters for Lorentz violation. The latter can be taken as the sum of the perturbative Hamiltonians for the particles comprising $W$ :

$$
h^{\prime}=\sum_{w} \sum_{N=1}^{N_{w}} \delta h_{w, N} .
$$

The perturbative Hamiltonian $\delta h_{w, N}$ for the $N$ th particle of type $w$ is of the same general form as $\delta h$ given in Eq. (4), except that allowance must be made for the possibility that the parameters for Lorentz violation depend on the particle species $w$. In what follows, this dependence is indicated by a superscript $w$ on the parameters $a_{\mu}, b_{\mu}, c_{\mu \nu}, d_{\mu \nu}, e_{\mu}, f_{\mu}$, $g_{\lambda \mu \nu}$, and $H_{\mu \nu}$.

The shift of an energy level induced by the Lorentz violation can be calculated as usual by taking the expectation value of the perturbative Hamiltonian in the appropriate unperturbed quantum state. For almost all experiments of interest here, the total angular momentum $\vec{F}$ of the atom or ion and its projection along the quantization axis are conserved to an excellent approximation. The quantization axis is typically determined by the orientation of an external magnetic field, and for simplicity we always define the $z$ direction in the laboratory frame as this quantization axis. Conservation of $\vec{F}$ and $F_{3}$ means that the corresponding quantum numbers $F$ and $m_{F}$ can be used to label a quantum state of $W$ as $\left|F, m_{F}\right\rangle$, so we proceed under this assumption. In fact, the rotational symmetry of one experiment of interest [3] is sufficiently broken by the applied (magnetic) field that $F$ cannot 
be taken as a good quantum number. However, in this case $\left|F, m_{F}\right\rangle$ can be replaced by $\left|I, m_{I}\right\rangle$ where $I$ is the quantum number for nuclear spin and $m_{I}$ is the quantum number for its projection along the quantization axis. This point is discussed further in the Appendix.

The perturbative energy shift of the state $\left|F, m_{F}\right\rangle$ due to Lorentz violations is given by $\left\langle F, m_{F}\left|h^{\prime}\right| F, m_{F}\right\rangle$. However, only certain parts of $h^{\prime}$ are relevant for this calculation because the properties of $h^{\prime}$ and of the states $\left|F, m_{F}\right\rangle$ constrain some terms to have zero expectation value. For example, since the relevant states of $W$ are all bound, $\langle\vec{p}\rangle=0$ for all states. More generally, the expectation value of any odd power of momentum $\vec{p}$ vanishes, and so all terms in $h^{\prime}$ proportional to an odd power of $\vec{p}$ are irrelevant for our purposes.

Additional constraints are provided by the rotation properties of the states $\left|F, m_{F}\right\rangle$. The expectation value of $h^{\prime}$ in a state $\left|F, m_{F}\right\rangle$ can be written as a linear combination of terms of the form $\left\langle F, m_{F}\left|T_{q}^{(r)}\right| F, m_{F}\right\rangle$, where $T_{q}^{(r)}$ represents the $q$ component of a spherical tensor operator of rank $r(q=$ $-r, \ldots, r)$. Note that individual terms in the linear combination with $r=0$ are irrelevant to clock-comparison experiments because they are rotationally invariant. The relevant terms are partially fixed by the Wigner-Eckart theorem [32]. This implies some terms vanish, including any with $q \neq 0$, and simplifies the structure of the surviving terms. Thus, each surviving term is the product of two factors, one being a ratio of Clebsch-Gordan coefficients and the other being an expectation value in the special state $\left|F, m_{F}=F\right\rangle$. Only the former depends on $m_{F}$.

Restricting attention only to terms in $h^{\prime}$ that generate nonzero contributions relevant to clock-comparison experiments, one finds spherical tensor operators only of rank 1 or of rank 2. Since these operators have definite and distinct properties under rotations, it is useful to introduce terminology distinguishing their contributions to energy-level shifts. We therefore define the multipolarity of an energy shift according to the rank of the tensor from which it originates. For example, a dipole energy shift is one arising from an expectation value of a tensor of rank 1, while a quadrupole energy shift is one arising from an expectation value of a tensor of rank 2. The Wigner-Eckart theorem implies that the energy-level shifts in $W$ can have multipolarities at most of order $2 F$. However, despite the generality of the theoretical framework, no leading-order octupole or higher-order energy shifts can emerge from the Hamiltonian $h^{\prime}$ because the tensor operators involved are all of rank 2 or less. Since monopole shifts may exist but are unobservable in clockcomparison experiments, only dipole and quadrupole energy shifts are relevant to the analysis here.

Implementing the above calculations, we find that the leading-order energy shift due to Lorentz violations of the state $\left|F, m_{F}\right\rangle$ of the atom or ion $W$ is a sum of two terms,

$$
\left\langle F, m_{F}\left|h^{\prime}\right| F, m_{F}\right\rangle=\hat{m}_{F} E_{d}^{W}+\widetilde{m}_{F} E_{q}^{W} .
$$

In this expression, $\hat{m}_{F}$ and $\widetilde{m}_{F}$ are ratios of Clebsch-Gordan coefficients arising from the application of the WignerEckart theorem and given by

$$
\hat{m}_{F}:=\frac{m_{F}}{F}, \quad \widetilde{m}_{F}:=\frac{3 m_{F}^{2}-F(F+1)}{3 F^{2}-F(F+1)} .
$$

The dipole and quadrupole energy shifts $E_{d}^{W}$ and $E_{q}^{W}$ are independent of $m_{F}$ and are given by

$$
\begin{aligned}
& E_{d}^{W}=\sum_{w}\left(\beta_{w} \widetilde{b}_{3}^{w}+\delta_{w} \widetilde{d}_{3}^{w}+\kappa_{w} \widetilde{g}_{d}^{w}\right), \\
& E_{q}^{W}=\sum_{w}\left(\gamma_{w} \widetilde{c}_{q}^{w}+\lambda_{w} \widetilde{g}_{q}^{w}\right)
\end{aligned}
$$

in terms of quantities to be defined below. The cartesian components in these and all subsequent expressions in this subsection refer to coordinates in the laboratory frame.

In Eq. (8), the various quantities with tildes are combinations of the parameters for Lorentz violation appearing in the nonrelativistic Hamiltonians for the component particles $w$ of $W$. These are the only parameter combinations that could in principle be bounded in clock-comparison experiments with ordinary matter. They are defined by

$$
\begin{aligned}
& \widetilde{b}_{3}^{w}:=b_{3}^{w}-m_{w} d_{30}^{w}+m_{w} g_{120}^{w}-H_{12}^{w}, \\
& \widetilde{c}_{q}^{w}:=m_{w}\left(c_{11}^{w}+c_{22}^{w}-2 c_{33}^{w}\right), \\
& \widetilde{d}_{3}^{w}:=m_{w} d_{03}^{w}+\frac{1}{2} m d_{30}^{w}-\frac{1}{2} H_{12}^{w}, \\
& \widetilde{g}_{d}^{w}:=m_{w}\left(g_{102}^{w}-g_{201}^{w}+g_{120}^{w}\right)-b_{3}^{w}, \\
& \widetilde{g}_{q}^{w}:=m_{w}\left(g_{101}^{w}+g_{202}^{w}-2 g_{303}^{w}\right) .
\end{aligned}
$$

Note that each of these is chosen to have dimensions of mass.

A calculation shows that the coefficients $\beta_{w}, \gamma_{w}, \delta_{w}$, $\kappa_{w}, \lambda_{w}$ appearing in Eq. (8) are linear combinations of expectation values in the special state $|F, F\rangle$ of certain operators appearing in the component nonrelativistic Hamiltonians for the particles $w$ comprising $W$ :

$$
\begin{aligned}
& \beta_{w}:=-\sum_{N=1}^{N_{w}}\left\langle\left[\sigma^{3}\right]_{w, N}\right\rangle, \\
& \gamma_{w}:=-\frac{1}{6 m_{w}^{2}} \sum_{N=1}^{N_{w}}\left\langle\left[p_{1}^{2}+p_{2}^{2}-2 p_{3}^{2}\right]_{w, N}\right\rangle, \\
& \delta_{w}:=\frac{1}{m_{w}^{2}} \sum_{N=1}^{N_{w}}\left\langle\left[p_{3} p_{j} \sigma^{j}\right]_{w, N}\right\rangle, \\
& \kappa_{w}:=\frac{1}{2 m_{w}^{2}} \sum_{N=1}^{N_{w}}\left\langle\left[p_{3} p_{j} \sigma^{j}-p_{j} p_{j} \sigma^{3}\right]_{w, N}\right\rangle, \\
& \lambda_{w}:=\frac{1}{2 m_{w}^{2}} \sum_{N=1}^{N_{w}}\left\langle\left[\left(p_{1} \sigma^{2}-p_{2} \sigma^{1}\right) p_{3}\right]_{w, N}\right\rangle .
\end{aligned}
$$


The subscript $w, N$ on each operator means that it acts on particle $N$ of type $w$. These coefficients are all dimensionless. Note that they depend on the specific atom or ion $W$.

An exact calculation of the values of the coefficients $\beta_{w}$, $\gamma_{w}, \delta_{w}, \kappa_{w}, \lambda_{w}$ is typically infeasible, in part due to the determining role played by the nuclear forces. Some comments about evaluating these coefficients can be found in Sec. II C. On dimensional grounds a nonzero value of $\beta_{w}$ is likely to be of order unity, while nonzero values of the other quantities are suppressed by a factor $K_{w}:=\left\langle p^{2}\right\rangle_{w} / m_{w}^{2}$, roughly given by $K_{p} \approx K_{n} \simeq 10^{-2}$ and $K_{e} \simeq 10^{-5}$.

\section{Comments on expectation values}

In this subsection, some aspects of the evaluation of the coefficients $\beta_{w}, \gamma_{w}, \delta_{w}, \kappa_{w}, \lambda_{w}$ defined in Eq. (10) are considered. Although exact results cannot typically be derived, partly because no exact treatment of nuclear forces is available, some statements based on symmetry arguments can be made despite the absence of precise knowledge of the electronic, nuclear, atomic, or ionic wave functions. For some special cases and within certain approximations, explicit results for the angular dependences of the coefficients in Eq. (10) can be obtained. Under suitable circumstances, some of the coefficients can be shown to vanish or to be independent of one or more of the particle species $w$.

Consider first the special case of an atom $W$ in which the electrons form a closed shell. To a good approximation, the expectation values in $|F, F\rangle$ appearing in Eq. (10) can then be replaced by expectation values in the state $|I, I\rangle$, where $I$ is the quantum number for the nuclear spin. Following the discussion in the previous subsection, the maximal multipolarity of the energy shifts is $2 I$ and only dipole and quadrupole energy shifts are observable. Thus, any nucleus with $I=0$ has no observable effects. A nucleus with $I=\frac{1}{2}$ may have nonzero dipole energy shifts $E_{d}^{W}$, but $E_{q}^{W}$ must vanish. All other nuclei may have both dipole and quadrupole shifts.

Further considerations based on Eq. (10) are needed to determine the specific dependence of the shifts on the proton and neutron parameters for Lorentz violation. One possibility is to work within a nuclear shell model [33-35]. Consider the special case where $W$ has a closed electronic shell, and where a single valence nucleon of one species lies outside closed proton and neutron shells. To a good approximation, the expectation values in $|F, F\rangle$ appearing in Eq. (10) can then be replaced by expectation values in the one-nucleon state $|j, j\rangle$, where $j=l \pm \frac{1}{2}$ is the total angular momentum of the valence nucleon $w$ and $l$ is the quantum number for its orbital angular momentum. This implies that the values of the coefficients in Eq. (10) can be nonzero only for this nucleon. After some calculation, we find for $j=l+\frac{1}{2}$ the result

$$
\begin{gathered}
\beta_{w}=-1, \quad \gamma_{w}=-\frac{1}{3} \frac{l}{(2 l+3)} \frac{\left\langle p^{2}\right\rangle_{w}}{m_{w}^{2}}, \\
\delta_{w}=\frac{1}{(2 l+3)} \frac{\left\langle p^{2}\right\rangle_{w}}{m_{w}^{2}},
\end{gathered}
$$

$$
\kappa_{w}=-\frac{(l+1)}{(2 l+3)} \frac{\left\langle p^{2}\right\rangle_{w}}{m_{w}^{2}}, \quad \lambda_{w}=0,
$$

while for $j=l-\frac{1}{2}$ we find

$$
\begin{gathered}
\beta_{w}=\frac{(2 l-1)}{(2 l+1)}, \quad \gamma_{w}=-\frac{1}{3} \frac{(l-1)}{(2 l+1)} \frac{\left\langle p^{2}\right\rangle_{w}}{m_{w}^{2}}, \\
\delta_{w}=-\frac{3(2 l-1)}{(2 l+1)(2 l+3)} \frac{\left\langle p^{2}\right\rangle_{w}}{m_{w}^{2}}, \\
\kappa_{w}=\frac{l(2 l-1)}{(2 l+1)(2 l+3)} \frac{\left\langle p^{2}\right\rangle_{w}}{m_{w}^{2}}, \quad \lambda_{w}=0 .
\end{gathered}
$$

In these expressions, the expectation value $\left\langle p^{2}\right\rangle_{w}$ is in the radial wave function.

Equations (11) and (12) hold in the general case when the electronic shell is closed and the nucleus can be described by the Schmidt model [36,37]. In this model, a single nucleon is assumed to carry the entire angular momentum of the nucleus. In the above equations, $j$ then becomes the nuclear spin $I$ and $l$ becomes the quantum number for the orbital angular momentum assigned to the single Schmidt nucleon. The above equations also apply to the electronic structure of an atom or ion in the special case where a single valence electron of orbital angular momentum $l$ and total angular momentum $j$ lies outside a closed shell.

More complex models can be used to gain further insight. As an explicit example, we consider ${ }^{7} \mathrm{Li}$, which was used in both of the original clock-comparison experiments $[1,2]$. An approximate wave function for the ${ }^{7} \mathrm{Li}$ nucleus can be found [38] using a model in which two of the protons and two of the neutrons combine to form an $\alpha$-particle core, leaving a single valence proton and two valence neutrons. The nuclear ground state has $\operatorname{spin} I=\frac{3}{2}$, so nonzero dipole and quadrupole energy shifts $E_{d}^{7 \mathrm{Li}}, E_{q}^{7} \mathrm{Li}$ are both possible in principle. Within the model, an approximation to the wave function of the nucleus is

$$
\psi^{7} \mathrm{Li}=C_{1}\left({ }^{1} D,{ }^{2} P\right)+C_{2}\left({ }^{1} S,{ }^{2} P\right),
$$

where $C_{1} \simeq 0.681$ and $C_{2} \simeq 0.732$ are constants. Each term in parentheses represents a multiparticle component wave function labeled as $\left({ }^{2 S_{n}+1} L_{n},{ }^{2 S_{p}+1} L_{p}\right)$, where $S_{p}, S_{n}$ are total spins and $L_{p}, L_{n}$ are total orbital angular momenta for the valence proton and neutrons.

This wave function can be used to calculate explicitly the coefficients appearing in Eq. (10), but the result provides relatively little insight. It is of more direct interest to note that the wave function (13) indicates that $S_{n}=0$ and $S_{p}$ $=\frac{1}{2}$. All the operators whose expectation values produce the dipole shift $E_{d}^{W}$ in Eq. (8) involve spin. It therefore follows within this model that $E_{d}^{7} \mathrm{Li}$ is independent of the neutron parameters for Lorentz violation but does depend on proton ones. However, the quadrupole shift $E_{q}^{W}$ in Eq. (8) involves the purely spatial operators appearing in the definition of $\gamma_{w}$ in Eq. (10). According to the wave function (13), this is 
expected to produce a nonzero contribution for both $\gamma_{n}{ }^{7 \mathrm{Li}}$ and $\gamma_{p}^{7}$ Li because terms with $L_{n}=2$ and $L_{p}=1$ appear.

This calculation can also be used to illustrate the dangers of relying on a particular model to deduce details of the origin of possible dipole or quadrupole shifts. A further refinement of the ${ }^{7} \mathrm{Li}$ nuclear wave function [38] produces an additional term $C_{3}\left({ }^{3} P,{ }^{2} P\right)$, with $C_{3} \simeq 0.1$. The extra term has $S_{n}=1$, indicating that $E_{d}^{7} \mathrm{Li}$ does depend on neutron parameters, although in a partially suppressed way. This calculation also shows that care is required in applying results from a simple nuclear shell model. The ground-state properties of any odd-mass nucleus $W$ with an even number of neutrons are supposed to be determined entirely by the protons, which would imply that both $E_{d}^{W}$ and $E_{q}^{W}$ are independent of neutron parameters. However, this is not strictly correct. A counterexample is provided by ${ }^{7} \mathrm{Li}$, as above. A similar issue arises for the ground-state properties of an oddmass nucleus with an even proton number, supposedly determined entirely by the neutrons. A counterexample here is provided by the ${ }^{9} \mathrm{Be}$ nucleus: using a multiparticle wave function [38], a calculation shows that $E_{q}^{{ }^{9} \mathrm{Be}}$ does in fact depend on proton parameters.

Despite the obstacles to definitive calculations of the coefficients in Eq. (10), some results holding under relatively mild assumptions can be obtained. For example, the WignerEckart theorem can be used to show that closed shells of particles make no contributions to either $E_{d}^{W}$ or $E_{q}^{W}$. A closed shell for some angular momentum $J$ has all substates $\left|J, m_{J}\right\rangle$ occupied, so the contribution $\Delta E_{J, r, q}$ from a closed shell to the energy shift caused by a spherical tensor operator $T_{q}^{(r)}$ of rank $r(q=-r, \ldots, r)$ is given by

$$
\Delta E_{J, r, q}=\sum_{m_{J}=-J}^{J}\left\langle J, m_{J}\left|T_{q}^{(r)}\right| J, m_{J}\right\rangle .
$$

By the Wigner-Eckart theorem, we find

$$
\Delta E_{J, r, q}=\delta_{q 0}\left\langle J, J\left|T_{0}^{(r)}\right| J, J\right\rangle \sum_{m_{J}=-J}^{J} \hat{c}_{J m_{J} r 0} .
$$

The coefficients $\hat{c}_{J m_{J} r 0}$ are ratios of Clebsch-Gordan coefficients. For the cases $r=1,2$ of interest we find $\hat{c}_{J m_{J} 10}=\hat{m}_{J}$ and $\hat{c}_{J m_{J} 20}=\widetilde{m}_{J}$, where $\hat{m}_{J}$ and $\widetilde{m}_{J}$ are given in Eq. (7). Explicit evaluation of the sum in Eq. (14) for these two cases then gives the claimed result, $\Delta E_{J, 1, q}=\Delta E_{J, 2, q}=0$.

More general cases, where $W$ has nontrivial electronic structure and contributions from multiple nucleons, could also be analyzed using the approaches in this subsection whenever a decomposition of the wave function $|F, F\rangle$ into a sum of multiparticle product wave functions provides an adequate description of the atom or ion. It then follows that the angular dependences of the quantities defined in Eq. (10) can in principle be calculated in terms of Clebsch-Gordan coefficients and the quantum numbers for the orbital and spin angular momenta of the component fermions of $W$.

\section{Geometry and time dependence}

The components of the parameters for Lorentz violation appearing in Eqs. (8) and (9) are defined in the laboratory frame. Since this frame rotates with the Earth, the components vary in time $t$ with a periodicity that depends on the Earth's sidereal rotation frequency $\Omega \simeq 2 \pi /(23 \mathrm{~h} 56 \mathrm{~min})$. Clock-comparison experiments typically bound the amplitude of the time variation of a transition frequency, which here is related to a difference between energy shifts of the form $\left\langle F, m_{F}\left|h^{\prime}\right| F, m_{F}\right\rangle$. Next, we determine the time dependence of the energy levels in terms of the parameters for Lorentz violation.

The first step is to introduce suitable bases of vectors for a nonrotating frame and for the laboratory frame. In what follows, the basis in the nonrotating frame is denoted $(\hat{X}, \hat{Y}, \hat{Z})$, while that in the laboratory frame is denoted $(\hat{x}, \hat{y}, \hat{z})$.

For the nonrotating frame, the rotation axis of the Earth provides a natural choice of $\hat{Z}$ axis. Astronomers define celestial equatorial coordinates [39] called declination and right ascension, which we use to fix the $\hat{X}$ and $\hat{Y}$ axes. The $\hat{Z}$ axis corresponds to declination $90^{\circ}$. We define $\hat{X}$ to have both declination and right ascension $0^{\circ}$, while $\hat{Y}$ has declination $0^{\circ}$ and right ascension $90^{\circ}$. Then, $(\hat{X}, \hat{Y}, \hat{Z})$ forms a righthanded orthonormal basis, with the basis vectors $\hat{X}$ and $\hat{Y}$ lying in the plane of the Earth's equator. To the extent that precession of the Earth's axis can be neglected [40], this basis is constant in time. It is also independent of any particular clock-comparison experiment.

For the laboratory frame, we take a natural definition of the $\hat{z}$ axis as the quantization axis of the atoms or ions involved in the specific experiment in question. This direction typically differs for different experiments, so the basis $(\hat{x}, \hat{y}, \hat{z})$ does too. The basis $(\hat{x}, \hat{y}, \hat{z})$ also varies in time, and the vector $\hat{z}$ precesses about $\hat{Z}$ with the Earth's sidereal frequency $\Omega$. A nonzero signal in a clock-comparison experiment preferentially requires that $\hat{z}$ not be parallel to $\hat{Z}$, since otherwise the time variation of the signal arises only from the precession of the Earth's axis and is heavily suppressed. In what follows, we therefore assume the angle $\chi \in(0, \pi)$ given by $\cos \chi=\hat{z} \cdot \hat{Z}$ is nonzero. We choose time $t=0$ such

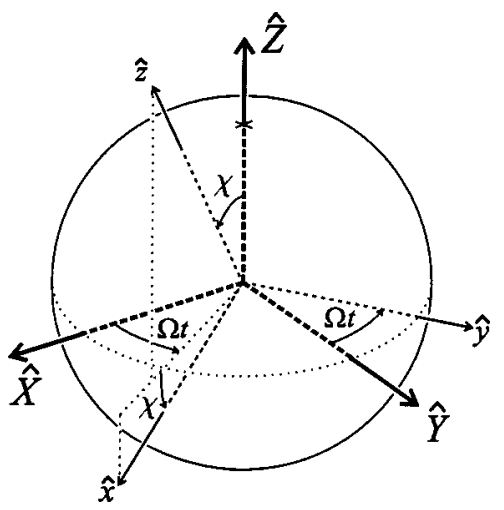

FIG. 1. Transformation of coordinates. 
that $\hat{z}(t=0)$ lies in the first quadrant of the $\hat{X}-\hat{Z}$ plane, and we define $\hat{x}$ to be perpendicular to $\hat{z}$ and to lie in the plane spanned by $\hat{z}$ and $\hat{Z}: \hat{x}:=\hat{z} \cot \chi-\hat{Z} \csc \chi$. Then, a righthanded orthonormal basis is obtained with the definition $\hat{y}:=\hat{z} \times \hat{x}$. With these choices, the $\hat{y}$ axis always lies in the plane of the Earth's equator and is thus perpendicular to $\hat{Z}$. Since the laboratory frame rotates about the $\hat{Z}$ axis with frequency $\Omega, \hat{y}$ coincides with $\hat{Y}$ once every (sidereal) day.

The two sets of basis vectors are shown in Fig. 1. To ease visualization, the basis $(\hat{x}, \hat{y}, \hat{z})$ has been translated from the surface of the globe to the center, so the origins of the two basis sets coincide. The rotation of the Earth is nonrelativistic to a good approximation, since a point on the Earth's equator moves with respect to the rotation axis at about $10^{-6}$ lightspeed. For most purposes the associated relativistic effects can therefore be ignored, and a nonrelativistic transformation between the two bases suffices. It is given by

$$
\left(\begin{array}{c}
\hat{x} \\
\hat{y} \\
\hat{z}
\end{array}\right)=\left(\begin{array}{ccc}
\cos \chi \cos \Omega t & \cos \chi \sin \Omega t & -\sin \chi \\
-\sin \Omega t & \cos \Omega t & 0 \\
\sin \chi \cos \Omega t & \sin \chi \sin \Omega t & \cos \chi
\end{array}\right)\left(\begin{array}{c}
\hat{X} \\
\hat{Y} \\
\hat{Z}
\end{array}\right)
$$

with the above basis definitions. This transformation can be used directly to obtain the time variation of the parameters for Lorentz violation.

To express the results in a relatively compact form, it is convenient to introduce nonrotating-frame analogues of the parameters in Eq. (9). We define

$$
\begin{aligned}
& \widetilde{b}_{J}::=b_{J}-m d_{J 0}+\frac{1}{2} m \epsilon_{J K L} g_{K L 0}-\frac{1}{2} \epsilon_{J K L} H_{K L}, \\
& \widetilde{c}_{Q}:=m\left(c_{X X}+c_{Y Y}-2 c_{Z Z}\right), \\
& \widetilde{c}_{Q, J}:=m\left(c_{J Z}+c_{Z J}\right), \quad J=X, Y, \\
& \widetilde{c}_{-}:=m\left(c_{X X}-c_{Y Y}\right), \quad \widetilde{c}_{X Y}:=m\left(c_{X Y}+c_{Y X}\right), \\
& \widetilde{d}_{J}:=m\left(d_{0 J}+d_{J 0}\right)-\frac{1}{2}\left(m d_{J 0}+\frac{1}{2} \varepsilon_{J K L} H_{K L}\right), \\
& \widetilde{g}_{D, J}:=m \varepsilon_{J K L}\left(g_{K 0 L}+\frac{1}{2} g_{K L 0}\right)-b_{J}, \\
& \widetilde{g}_{Q}:=m\left(g_{X 0 X}+g_{Y 0 Y}-2 g_{Z 0 Z}\right), \\
& \widetilde{g}_{Q, J}:=m\left(g_{J 0 Z}+g_{Z 0 J}\right), \quad J=X, Y, \\
& \widetilde{g}_{-}:=m\left(g_{X 0 X}-g_{Y 0 Y}\right), \quad \widetilde{g}_{X Y}:=m\left(g_{X 0 Y}+g_{Y 0 X}\right) .
\end{aligned}
$$

Here, spatial indices in the nonrotating frame are denoted by $J=X, Y, Z$ except where indicated, the time index is denoted 0 , and $\epsilon_{J K L}$ is the nonrotating-frame analogue of $\epsilon_{j k l}$ obeying $\epsilon_{X Y Z}=+1$. The label $w$ is suppressed for simplicity.

With these definitions, the transformation matrix in Eq. (16) can be used to express the time dependence of the parameters for Lorentz violation in the laboratory frame:

$$
\begin{aligned}
\widetilde{b}_{3}= & \widetilde{b}_{Z} \cos \chi+\widetilde{b}_{X} \sin \chi \cos \Omega t+\widetilde{b}_{Y} \sin \chi \sin \Omega t, \\
\widetilde{c}_{q}= & \widetilde{c}_{Q}\left(\frac{3}{2} \cos ^{2} \chi-\frac{1}{2}\right)-\frac{3}{2} \widetilde{c}_{Q, X} \sin 2 \chi \cos \Omega t \\
& -\frac{3}{2} \widetilde{c}_{Q, Y} \sin 2 \chi \sin \Omega t-\frac{3}{2} \widetilde{c}_{-} \sin ^{2} \chi \cos 2 \Omega t \\
& -\frac{3}{2} \widetilde{c}_{X Y} \sin ^{2} \chi \sin 2 \Omega t, \\
\widetilde{d}_{3}= & \widetilde{d}_{Z} \cos \chi+\widetilde{d}_{X} \sin \chi \cos \Omega t+\widetilde{d}_{Y} \sin \chi \sin \Omega t, \\
\widetilde{g}_{d}= & \widetilde{g}_{D, Z} \cos \chi+\widetilde{g}_{D, X} \sin \chi \cos \Omega t+\widetilde{g}_{D, Y} \sin \chi \sin \Omega t, \\
\widetilde{g}_{q}= & \widetilde{g}_{Q}\left(\frac{3}{2} \cos ^{2} \chi-\frac{1}{2}\right)-\frac{3}{2} \widetilde{g}_{Q, X} \sin 2 \chi \cos \Omega t \\
& -\frac{3}{2} \widetilde{g}_{Q, Y} \sin 2 \chi \sin \Omega t-\frac{3}{2} \widetilde{g}_{-} \sin ^{2} \chi \cos 2 \Omega t \\
& -\frac{3}{2} \widetilde{g}_{X Y} \sin ^{2} \chi \sin 2 \Omega t .
\end{aligned}
$$

Note that $\widetilde{b}_{3}, \widetilde{d}_{3}$, and $\widetilde{g}_{d}$ involve constant pieces and ones varying with the sidereal frequency $\Omega$, while the others also have terms varying with the semisidereal frequency $2 \Omega$. Note also that the parameters $\widetilde{b}_{Z}, \widetilde{c}_{Q}, \widetilde{d}_{Z}, \widetilde{g}_{D, Z} \widetilde{g}_{Q}$ appear only in time-independent terms, and they therefore are unconstrained by clock-comparison experiments.

Substituting the above into the expression (6) for the energy-level shift gives

$$
\begin{aligned}
\left\langle F, m_{F}\left|h^{\prime}\right| F, m_{F}\right\rangle= & E_{0}+E_{1 X} \cos \Omega t+E_{1 Y} \sin \Omega t \\
& +E_{2 X} \cos 2 \Omega t+E_{2 Y} \sin 2 \Omega t .
\end{aligned}
$$

The energy $E_{0}$ is constant in time and is therefore irrelevant for clock-comparison experiments. The four other energies are defined by

$$
\begin{aligned}
E_{1 X}:= & \hat{m}_{F} \sin \chi \sum_{w}\left(\beta_{w} \widetilde{b}_{X}^{w}+\delta_{w} \widetilde{d}_{X}^{w}+\kappa_{w} \widetilde{g}_{D, X}^{w}\right) \\
& -\frac{3}{2} \widetilde{m}_{F} \sin 2 \chi \sum_{w}\left(\gamma_{w} \widetilde{c}_{Q, X}^{w}+\lambda_{w} \widetilde{g}_{Q, X}^{w}\right), \\
E_{1 Y}:= & \hat{m}_{F} \sin \chi \sum_{w}\left(\beta_{w} \widetilde{b}_{Y}^{w}+\delta_{w} \widetilde{d}_{Y}^{w}+\kappa_{w} \widetilde{g}_{D, Y}^{w}\right) \\
& -\frac{3}{2} \widetilde{m}_{F} \sin 2 \chi \sum_{w}\left(\gamma_{w} \widetilde{c}_{Q, Y}^{w}+\lambda_{w} \widetilde{g}_{Q, Y}^{w}\right), \\
E_{2 X}:= & -\frac{3}{2} \widetilde{m}_{F} \sin ^{2} \chi \sum_{w}\left(\gamma_{w} \widetilde{c}_{-}^{w}+\lambda_{w} \widetilde{g}_{-}^{w}\right), \\
E_{2 Y}:= & -\frac{3}{2} \widetilde{m}_{F} \sin ^{2} \chi \sum_{w}\left(\gamma_{w} \widetilde{c}_{X Y}^{w}+\lambda_{w} \widetilde{g}_{X Y}^{w}\right) .
\end{aligned}
$$

For clock-comparison experiments, the signal is typically a time variation in a frequency. In the context of the present 
theoretical framework, this is determined by the difference between two energy-level shifts of the form Eq. (19).

The reader should note that the component of the shift in Eq. (19) varying with the sidereal frequency $\Omega$ is determined by operators producing both dipole and quadrupole energy shifts. The issue of the multipolarity of the energy shift, which is governed by the rotation properties of the Lorentzviolating perturbations in the laboratory frame, is somewhat different from the issue of the periodicity of the signals in clock-comparison experiments, which is governed also by factors associated with the Earth's rotation. The relatively simple correspondences sometimes found in the literature between the multipolarity of the energy shift and the periodicity of the signal or the effects on the spectrum are invalid in the general case [41].

The use of the nonrelativistic transformation (16) between the nonrotating and laboratory frames means that contributions from nonrotating-frame time components of the parameters for Lorentz violation are absent. In a more exact treatment, these quantities would be present but suppressed by a factor of order $10^{-6}$. For example, $\widetilde{b}_{3}$ strictly also depends slightly on the nonrotating-frame timelike component $b_{0}$. This means that some bounds on certain time components of the parameters could in principle be obtained. However, these bounds would be much weaker than the ones considered here. Moreover, an accurate treatment would also require inclusion of some of the other subleading effects mentioned at the beginning of this section.

\section{APPLICATION}

This section applies the theoretical framework of the previous section to existing and future clock-comparison experiments. The limits attained in the original experiments of Hughes et al. [1] and Drever [2] have been improved by many orders of magnitude in recent years. In the first part of this section, we focus our attention on results from the clockcomparison experiments performed by Prestage et al. [3], Lamoreaux et al. [4], Chupp et al. [5], and Berglund et al. [6]. The theory presented in Sec. II can be used to extract from each of these experiments one or more bounds on combinations of parameters for Lorentz violation. In the second part of this section, we present some considerations relevant to possible future experiments.

For purposes of discussion, it is useful to format all the bounds in a unified way. In effect, each experiment observes the frequency of one atomic or ionic species $A$ relative to a reference frequency in another species $B$, producing one or more bounds on possible sidereal or fractional-sidereal variations as the Earth rotates. Within the present framework, the effect of Lorentz violations on these frequencies can be deduced from the perturbative energy shifts given in Eq. (19). Some comments about this procedure are provided in the Appendix.

We find that each bound from each experiment fits one of the following forms:

$$
\begin{aligned}
& \mid \sum_{w}\left[u_{0}^{A}\left(\beta_{w}^{A} \widetilde{b}_{X}^{w}+\delta_{w}^{A} \widetilde{d}_{X}^{w}+\kappa_{w}^{A} \widetilde{g}_{D, X}^{w}\right)+u_{1}^{A}\left(\gamma_{w}^{A} \widetilde{c}_{Q, X}^{w}+\lambda_{w}^{A} \widetilde{g}_{Q, X}^{w}\right)\right] \\
& -\mathrm{v} \sum_{w}\left[u_{0}^{B}\left(\beta_{w}^{B} \widetilde{b}_{X}^{w}+\delta_{w}^{B} \widetilde{d}_{X}^{w}+\kappa_{w}^{B} \widetilde{g}_{D, X}^{w}\right)\right. \\
& \left.+u_{1}^{B}\left(\gamma_{w}^{B} \widetilde{c}_{Q, X}^{w}+\lambda_{w}^{B} \widetilde{g}_{Q, X}^{w}\right)\right] \mid \leq 2 \pi \varepsilon_{1, X}, \\
& \mid \sum_{w}\left[u_{0}^{A}\left(\beta_{w}^{A} \widetilde{b}_{Y}^{w}+\delta_{w}^{A} \widetilde{d}_{Y}^{w}+\kappa_{w}^{A} \widetilde{g}_{D, Y}^{w}\right)+u_{1}^{A}\left(\gamma_{w}^{A} \widetilde{c}_{Q, Y}^{w}+\lambda_{w}^{A} \widetilde{g}_{Q, Y}^{w}\right)\right] \\
& -\mathrm{v} \sum_{w}\left[u_{0}^{B}\left(\beta_{w}^{B} \widetilde{b}_{Y}^{w}+\delta_{w}^{B} \widetilde{d}_{Y}^{w}+\kappa_{w}^{B} \widetilde{g}_{D, Y}^{w}\right)\right. \\
& \left.\quad+u_{1}^{B}\left(\gamma_{w}^{B} \widetilde{c}_{Q, Y}^{w}+\lambda_{w}^{B} \widetilde{g}_{Q, Y}^{w}\right)\right] \mid \leq 2 \pi \varepsilon_{1, Y}, \\
& \left|\sum_{w} u_{2}^{A}\left(\gamma_{w}^{A} \widetilde{c}_{-}^{w}+\lambda_{w}^{A} \widetilde{g}_{-}^{w}\right)-\mathrm{v} \sum_{w} u_{2}^{B}\left(\gamma_{w}^{B} \widetilde{c}_{-}^{w}+\lambda_{w}^{B} \widetilde{g}_{-}^{w}\right)\right| \\
& \quad \leq 2 \pi \varepsilon_{2,-}, \\
& \left|\sum_{w} u_{2}^{A}\left(\gamma_{w}^{A} \widetilde{c}_{X Y}^{w}+\lambda_{w}^{A} \widetilde{g}_{X Y}^{w}\right)-v \sum_{w} u_{2}^{B}\left(\gamma_{w}^{B} \widetilde{c}_{X Y}^{w}+\lambda_{w}^{B} \widetilde{g}_{X Y}^{w}\right)\right| \\
& \leq 2 \pi \varepsilon_{2, X Y} .
\end{aligned}
$$

Here, the coefficients $u_{0}, u_{1}, u_{2}$, and $v$ contain the dependences on quantities such as $\hat{m}_{F}, \widetilde{m}_{F}, \chi$, and gyromagnetic ratios. For example, if an atom or ion $W$ undergoes a transition $\left|F, m_{F}^{\prime}\right\rangle \rightarrow\left|F, m_{F}\right\rangle$, then $u_{0}=\left(\hat{m}_{F}^{\prime}-\hat{m}_{F}\right) \sin \chi, \quad u_{1}=$ $-\frac{3}{2}\left(\widetilde{m}_{F}^{\prime}-\widetilde{m}_{F}\right) \sin 2 \chi$, and $u_{2}=-\frac{3}{2}\left(\widetilde{m}_{F}^{\prime}-\widetilde{m}_{F}\right) \sin ^{2} \chi$. The parameter $\mathbf{V}=g_{A} / g_{B}$ is the ratio of gyromagnetic ratios for the species $A$ and $B$. Also, the experimental bounds on the amplitudes of frequency shifts are denoted by $\varepsilon_{1, X}, \varepsilon_{1, Y}, \varepsilon_{2,-}$, $\varepsilon_{2, X Y}$, corresponding to sidereal or semisidereal variations as $\cos \Omega t, \sin \Omega t, \cos 2 \Omega t, \sin 2 \Omega t$, respectively. The other quantities are defined earlier in the text. For example, the factors $\beta_{w}, \gamma_{w}, \delta_{w}, \kappa_{w}$, and $\lambda_{w}$ are those given in Eq. (10), with subscripts according to the particle species and superscripts according to the atomic or ionic species. The components of the parameters for Lorentz violation are those for the nonrotating frame $(\hat{X}, \hat{Y}, \hat{Z})$. Note that the second of the equations is the same as the first but with the replacement $X \rightarrow Y$, reflecting the arbitrariness of the choice of $X$ and $Y$ axes in the $X Y$ plane. The form of the third and fourth of the above equations also reflects this arbitrariness.

The values of all relevant coefficients for each of the experiments we consider are summarized in Table I. The first few rows of this table identify the experiment and provide information about the atoms or ions used. We denote the nuclear spin by $I$, the proton number by $Z$, and the neutron number by $N$. The nucleon determining the ground-state properties of the nucleus according to the nuclear Schmidt model [36,37] is specified, along with its assignment of orbital and total angular momenta. Fifteen rows are devoted to the values of the coefficients $\beta_{w}, \gamma_{w}, \delta_{w}, \kappa_{w}$, and $\lambda_{w}$ for 
TABLE I. Coefficients for the bounds (21) for various experiments.

\begin{tabular}{|c|c|c|c|c|c|c|c|c|}
\hline & \multicolumn{2}{|c|}{ Prestage et al. [3] } & \multicolumn{2}{|c|}{ Lamoreaux et al. [4] } & \multicolumn{2}{|c|}{ Chupp et al. [5] } & \multicolumn{2}{|c|}{ Berglund et al. [6] } \\
\hline & $\begin{array}{c}A \\
{ }^{9} \mathrm{Be}^{+}\end{array}$ & $\begin{array}{c}B \\
{ }^{1} \mathrm{H}\end{array}$ & $\begin{array}{c}A \\
{ }^{201} \mathrm{Hg}\end{array}$ & $\begin{array}{c}B \\
{ }^{199} \mathrm{Hg}\end{array}$ & $\begin{array}{c}A \\
{ }^{21} \mathrm{Ne}\end{array}$ & $\begin{array}{c}B \\
{ }^{3} \mathrm{He}\end{array}$ & $\begin{array}{c}A \\
{ }^{199} \mathrm{Hg}\end{array}$ & $\begin{array}{c}B \\
{ }^{133} \mathrm{Cs}\end{array}$ \\
\hline$I$ & $3 / 2$ & $1 / 2$ & $3 / 2$ & $1 / 2$ & $3 / 2$ & $1 / 2$ & $1 / 2$ & $7 / 2$ \\
\hline$Z$ & 4 & 1 & 80 & 80 & 10 & 2 & 80 & 55 \\
\hline$N$ & 5 & 0 & 121 & 119 & 11 & 1 & 119 & 78 \\
\hline $\begin{array}{l}\text { Schmidt } \\
\text { nucleon }\end{array}$ & $\begin{array}{c}p_{3 / 2} \\
n\end{array}$ & $\begin{array}{c}s_{1 / 2} \\
p\end{array}$ & $\begin{array}{c}p_{3 / 2} \\
n\end{array}$ & $\begin{array}{c}p_{1 / 2} \\
n\end{array}$ & $\begin{array}{c}p_{3 / 2} \\
n\end{array}$ & $\begin{array}{c}s_{1 / 2} \\
n\end{array}$ & $\begin{array}{c}p_{1 / 2} \\
n\end{array}$ & $\begin{array}{c}g_{7 / 2} \\
p\end{array}$ \\
\hline$\overline{\beta_{p}}$ & {$[0]$} & - & {$[0]$} & {$[0]$} & - & - & {$[0]$} & {$\left[\frac{7}{9}\right]$} \\
\hline$\gamma_{p}$ & {$[0]$} & - & {$[0]$} & 0 & {$[0]$} & 0 & - & - \\
\hline$\delta_{p}$ & {$[0]$} & - & {$[0]$} & {$[0]$} & - & - & {$[0]$} & {$\left[-\frac{7}{33} K_{p}\right]$} \\
\hline$\kappa_{p}$ & {$[0]$} & - & {$[0]$} & {$[0]$} & - & - & {$[0]$} & {$\left[\frac{28}{99} K_{p}\right]$} \\
\hline$\lambda_{p}$ & {$[0]$} & - & {$[0]$} & 0 & {$[0]$} & 0 & - & - \\
\hline$\beta_{n}$ & {$[-1]$} & - & {$[-1]$} & {$[1 / 3]$} & - & - & {$[1 / 3]$} & {$[0]$} \\
\hline$\gamma_{n}$ & {$\left[-\frac{1}{15} K_{n}\right]$} & - & {$\left[-\frac{1}{15} K_{n}\right]$} & 0 & {$\left[-\frac{1}{15} K_{n}\right]$} & 0 & - & - \\
\hline$\delta_{n}$ & {$\left[\frac{1}{5} K_{n}\right]$} & - & {$\left[\frac{1}{5} K_{n}\right]$} & {$\left[-\frac{1}{5} K_{n}\right]$} & - & - & {$\left[-\frac{1}{5} K_{n}\right]$} & {$[0]$} \\
\hline$\kappa_{n}$ & {$\left[-\frac{2}{5} K_{n}\right]$} & - & {$\left[-\frac{2}{5} K_{n}\right]$} & {$\left[\frac{1}{15} K_{n}\right]$} & - & - & {$\left[\frac{1}{15} K_{n}\right]$} & {$[0]$} \\
\hline$\lambda_{n}$ & [0] & - & [0] & 0 & {$[0]$} & 0 & - & - \\
\hline$\beta_{e}$ & - & - & 0 & 0 & - & - & 0 & {$[-1]$} \\
\hline$\gamma_{e}$ & - & - & 0 & 0 & 0 & 0 & - & - \\
\hline$\delta_{e}$ & - & - & 0 & 0 & - & - & 0 & {$\left[\frac{1}{3} K_{e}\right]$} \\
\hline$\kappa_{e}$ & - & - & 0 & 0 & - & - & 0 & {$\left[-\frac{1}{3} K_{e}\right]$} \\
\hline$\lambda_{e}$ & - & - & 0 & 0 & 0 & 0 & - & - \\
\hline$u_{0}$ & -0.61 & - & $\frac{2}{3}$ & 2 & - & - & 2 & $\frac{1}{4}$ \\
\hline$u_{1}$ & 2.16 & - & 0 & 0 & - & - & 0 & 0 \\
\hline$u_{2}$ & -2.54 & - & -3 & 0 & -3 & - & - & - \\
\hline v & \multicolumn{2}{|l|}{-} & \multicolumn{2}{|c|}{-0.37} & \multicolumn{2}{|l|}{-} & \multicolumn{2}{|c|}{$2.2 \times 10^{-3}$} \\
\hline $\begin{array}{l}\varepsilon_{1, X}, \varepsilon_{1, Y} \\
\varepsilon_{2,-}, \varepsilon_{2, X Y}\end{array}$ & \multicolumn{2}{|c|}{$\begin{array}{l}\simeq 100 \mu \mathrm{Hz} \\
\simeq 100 \mu \mathrm{Hz}\end{array}$} & \multicolumn{2}{|c|}{$\begin{array}{l}\simeq 1 \mu \mathrm{Hz} \\
\simeq 1 \mu \mathrm{Hz}\end{array}$} & \multicolumn{2}{|c|}{$\begin{array}{l}\text { no bound } \\
\simeq 1 \mu \mathrm{Hz}\end{array}$} & \multicolumn{2}{|c|}{$\begin{array}{l}\simeq 100 \mathrm{nHz} \\
\text { no bound }\end{array}$} \\
\hline $\begin{array}{l}\text { Sensitivity } \\
(J=X, Y)\end{array}$ & \multicolumn{2}{|c|}{$\begin{array}{l}{\left[\tilde{b}_{J}^{n}, \widetilde{d}_{J}^{n}, \widetilde{g}_{D, J}^{n},\right.} \\
\left.\widetilde{c}_{Q, J}^{n}, \widetilde{c}_{-}^{n}, \widetilde{c}_{X Y}^{n}\right]\end{array}$} & \multicolumn{2}{|c|}{$\left[\widetilde{b}_{J}^{n}, \widetilde{d}_{J}^{n}, \widetilde{g}_{D, J}^{n}, \widetilde{c}_{-}^{n}, \widetilde{c}_{X Y}^{n}\right]$} & \multicolumn{2}{|c|}{$\left[\widetilde{c}_{-}^{n}, \widetilde{c}_{X Y}^{n}\right]$} & \multicolumn{2}{|c|}{$\begin{array}{c}{\left[\widetilde{b}_{J}^{p}, \widetilde{d}_{J}^{p}, \widetilde{g}_{D, J}^{p}, \widetilde{b}_{J}^{n}, \widetilde{d}_{J}^{n}, \widetilde{g}_{D, J}^{n},\right.} \\
\left.\tilde{b}_{J}^{e}, \widetilde{d}_{J}^{e}, \widetilde{g}_{D, J}^{p}\right]\end{array}$} \\
\hline
\end{tabular}

each atom or ion. In these rows, a dash indicates that the (possibly nonzero) coefficient is irrelevant for the experiment. Values in brackets are results obtained within the Schmidt model, with $K_{w}$ defined by $K_{w}:=\left\langle p^{2}\right\rangle_{w} / m_{w}^{2}$ as in Sec. II B. These values are to be trusted only where the model is known to give reliable results. Any zero values in brackets are probably unreliable because they are likely to be nonzero in more realistic nuclear models. Zero values without brackets are a consequence of the Wigner-Eckart theorem applied to closed shells, according to the discussion at the end of Sec. II C, and therefore depend on fewer assumptions. The following four rows specify the coefficients $u_{0}$, $u_{1}, u_{2}$, v. A dash indicates the coefficient is irrelevant for the experiment. Two rows provide approximate values for the experimental sidereal and semisidereal bounds obtained. In the final row, a list is provided of the parameters for
Lorentz violation constrained by the experiment according to this analysis.

The table reveals a crucial point: the published experiments are all inequivalent, in the sense that they bound different linear combinations of parameters for Lorentz violation. In some cases, such as the experiments of Chupp et al. [5] and Berglund et al. [6], there is no overlap at all among the set of parameters for Lorentz violation appearing in the bounds. In other cases, such as the experiments of Prestage et al. [3] and Lamoreaux et al. [4], there is substantial overlap among the parameters for Lorentz violation involved but they appear in different linear combinations.

In the context of the Schmidt model, the Prestage et al., Lamoreaux et al., and Chupp et al. experiments are sensitive only to (different sets of) parameters for Lorentz violation involving the neutron, $w \equiv n$. In contrast, the experiment of 
Berglund et al. involves all three particle species because the ${ }^{199} \mathrm{Hg}$ atom is sensitive to parameters for Lorentz violation with $w \equiv n$ and the ${ }^{133} \mathrm{Cs}$ atom is sensitive to ones with $w$ $\equiv p, e$. None of the experiments considered place any bound on the quantities $\widetilde{c}_{Q, J}^{e}, \widetilde{c}_{-}^{e}, \widetilde{c}_{X Y}^{e}, \widetilde{g}_{Q, J}^{e}, \widetilde{g}_{-}^{e}, \widetilde{g}_{X Y}^{e}$, while within the Schmidt model no bounds are placed on $\widetilde{c}_{Q, J}^{p}$, $\widetilde{c}_{X Y}^{p}, \widetilde{c}_{-}^{p}, \widetilde{g}_{Q, J}^{p}, \widetilde{g}_{X Y}^{p}, \widetilde{g}_{-}^{p}, \widetilde{g}_{Q, J}^{n}, \widetilde{g}_{X Y}^{n}, \widetilde{g}_{-}^{n}$. Note that some of these quantities can be bounded in other kinds of experiments [18,19].

A more accurate nuclear model would be likely to introduce dependence on parameters with $w \equiv p$ for all atoms and ions in these experiments except the $H$ maser used by Prestage et al. and the ${ }^{3} \mathrm{He}$ reference used by Chupp et al. Thus, the true bounds from all four experiments are likely to involve parameters for more than one species $w$. For the experiment of Berglund et al., the true dependence on parameters with $w \equiv p$ might even come primarily from the ${ }^{199} \mathrm{Hg}$ atom because the contribution from the ${ }^{133} \mathrm{Cs}$ atom is suppressed by its relatively large gyromagnetic ratio, reflected in Table I by the small size of the coefficient $v$.

The numerical values of the bounds obtained in all these experiments are impressive and represent sensitivity to Planck-scale physics. In contrast, the relatively complicated form of the linear combinations bounded and the theoretical issues involved in accurately determining the various coefficients make it difficult to establish definitively which portion of the parameter space is in fact being excluded. It is therefore of some interest to speculate about the implications of these experiments under different scenarios that lead to theoretically cleaner bounds.

One plausible assumption is that effects from the first atomic or ionic species $A$ would be unlikely to cancel completely the effects from the second species $B$ in Eq. (21). This assumption would permit (perhaps numerically weaker) bounds to be placed on somewhat simpler combinations of parameters. A stronger extension of this assumption might also be adopted to the effect that for a single species exact cancellations are unlikely among different terms in the sums appearing in Eq. (21). If this stronger assumption also holds, then for a given experimental bound the numerical value could be applied to each term in the sum, yielding plausible (but not definite) constraints on each of the parameters for Lorentz violation appearing in Eq. (21).

To gain some insight into the implications of these assumptions, one can examine the bounds that would follow within the additional approximation of the Schmidt model. Then, the sole experiment bounding electron or proton parameters for Lorentz violation is that of Berglund et al., which constrains only $\widetilde{b}_{J}^{w}, \widetilde{d}_{J}^{w}$, and $\widetilde{g}_{D, J}^{w}$ for $J=X, Y$. Also within these assumptions, the earlier experiments of Prestage et al., Lamoreaux et al., and Chupp et al. are insensitive to electron or proton parameters for Lorentz violation, but instead they have sensitivity to neutron parameters beyond the ones constrained by Berglund et al. For example, the experiment of Prestage et al. is the only one that constrains $\widetilde{c}_{Q, J}^{n}$.

Within these strong (and questionable) assumptions, approximate numerical bounds can be obtained by using dimensional estimates for the quantities $K_{w}$. As in Sec. II B,
TABLE II. Crude order-of-magnitude bounds in GeV on parameters for Lorentz violation.

\begin{tabular}{|c|c|c|c|c|}
\hline Expt. & [3] & [4] & {$[5]$} & {$[6]$} \\
\hline$\tilde{b}_{J}^{p}$ & $\star$ & $\star$ & - & {$\left[10^{-27}\right]$} \\
\hline$\widetilde{c}_{Q, J}^{p}$ & $\star$ & - & - & - \\
\hline$\tilde{c}_{-}^{p}$ & $\star$ & $\star$ & $\star$ & - \\
\hline$\tilde{c}_{X Y}^{p}$ & $\star$ & $\star$ & $\star$ & - \\
\hline$\tilde{d}_{J}^{p}$ & $\star$ & $\star$ & - & {$\left[10^{-25}\right]$} \\
\hline$\tilde{g}_{D, J}^{p}$ & $\star$ & $\star$ & - & {$\left[10^{-25}\right]$} \\
\hline$\widetilde{g}_{Q, J}^{p}$ & $\star$ & - & - & - \\
\hline$\tilde{g}_{-}^{\tilde{p}}$ & $\star$ & $\star$ & $\star$ & - \\
\hline$\tilde{g}_{X Y}^{p}$ & $\star$ & $\star$ & $\star$ & - \\
\hline$\widetilde{b}_{J}^{n}$ & {$\left[10^{-27}\right]$} & {$\left[10^{-29}\right]$} & - & {$\left[10^{-30}\right]$} \\
\hline$\widetilde{c}_{Q, J}^{n}$ & {$\left[10^{-25}\right]$} & - & - & - \\
\hline$\tilde{c}_{-}^{n}$ & {$\left[10^{-25}\right]$} & {$\left[10^{-27}\right]$} & {$\left[10^{-27}\right]$} & - \\
\hline$\widetilde{c}_{X Y}^{n}$ & {$\left[10^{-25}\right]$} & {$\left[10^{-27}\right]$} & {$\left[10^{-27}\right]$} & - \\
\hline$\widetilde{d}_{J}^{n}$ & {$\left[10^{-25}\right]$} & {$\left[10^{-26}\right]$} & - & {$\left[10^{-28}\right]$} \\
\hline$\widetilde{g}_{D, J}^{n}$ & {$\left[10^{-25}\right]$} & {$\left[10^{-27}\right]$} & - & {$\left[10^{-28}\right]$} \\
\hline$\widetilde{g}_{Q, J}^{n}$ & $\star$ & - & - & - \\
\hline$\widetilde{g}_{-}^{n}$ & $\star$ & $\star$ & $\star$ & - \\
\hline$\tilde{g}_{X Y}^{n}$ & $\star$ & $\star$ & $\star$ & - \\
\hline$\tilde{b}_{J}^{e}$ & - & - & - & {$\left[10^{-27}\right]$} \\
\hline$\widetilde{c}_{Q, J}^{e}$ & - & - & - & - \\
\hline$\widetilde{c}_{-}^{e}$ & - & - & - & - \\
\hline$\widetilde{c}_{X Y}^{e}$ & - & - & - & - \\
\hline$\tilde{d}_{J}^{e}$ & - & - & - & {$\left[10^{-22}\right]$} \\
\hline$\widetilde{g}_{D, J}^{e}$ & - & - & - & {$\left[10^{-22}\right]$} \\
\hline$\tilde{g}_{Q, J}^{e}$ & - & - & - & - \\
\hline$\tilde{g}_{-}^{e}$ & - & - & - & - \\
\hline$\widetilde{g}_{X Y}^{e}$ & - & - & - & - \\
\hline
\end{tabular}

we take the crude values $K_{p} \approx K_{n} \simeq 10^{-2}$ and $K_{e} \simeq 10^{-5}$. Table II presents the results of this estimate. In a given row, numerical values in brackets are estimated order-ofmagnitude bounds in $\mathrm{GeV}$ obtained within the Schmidt model assuming that all the parameters for Lorentz violation are zero except for the one specific to that row. The star indicates that no experimental bound is placed according to the Schmidt model but that a bound probably would emerge from a more realistic nuclear model. A dash indicates insensitivity to the specified parameter for Lorentz violation. The values obtained represent bounds on different parameters for Lorentz violation varying over about eight orders of magnitude, with the sharpest being a constraint on $\widetilde{b}_{J}^{n}$ of about $10^{-30} \mathrm{GeV}$. Although the approximations made imply that the specific numerical bounds listed in Table II are unreliable, in certain cases perhaps within several orders of magnitude, they nonetheless provide a gauge of the present impressive sensitivity of these experiments to the relevant parameters for Lorentz violation.

Still another speculation one might entertain, in addition to the above assumptions, is to suppose that cancellations are unlikely within each of the linear combinations in Eq. (17). 
If this were valid, then one could apply the numerical experimental bounds to deduce constraints on the original parameters for Lorentz violation appearing in the QED and standard-model extensions instead of merely constraining some of the combinations given in Eq. (17). However, this assumption is difficult to justify because it is likely that the various parameters in the QED extension are related through the underlying theory, perhaps in a relatively simple way, so significant cancellations may well occur. For example, a simple relation among certain parameters for CPT and Lorentz violation is known to occur in an anomaly-free underlying theory, and in this case it indeed eliminates the sensitivity of some experiments (on cosmological birefringence) to certain parameters for Lorentz violation $[8,23]$.

Despite the relatively complicated form of the bounds (21) and the theoretical issues in calculating the associated coefficients, the exceptional precision attained makes clockcomparison experiments of great interest. In principle, from a theoretical perspective a particularly attractive type of bound would be one that is both calculable and clean. Here, calculable refers to the reliability with which the various coefficients can be theoretically established, and clean refers to the number of different parameters for Lorentz violation involved in the bound (the fewer the cleaner). It is interesting to examine the extent to which calculable and clean bounds from clock-comparison experiments are theoretically possible.

For a bound to be calculable with the methods adopted here, reliable wave functions would need to be obtained for the atoms or ions used in the experiment. The complexity of nuclear matter typically makes this a challenging task. One possibility is to consider atoms or ions involving very few particles, so that a detailed calculation has an improved chance of accuracy.

Among the simplest substances is hydrogen. The welldeveloped theoretical understanding of the hydrogen wave function makes it a prime candidate for a substance that would produce a calculable bound. Various experiments with hydrogen atoms and ions $\left(\mathrm{H}^{-}\right.$and the proton) and with antihydrogen have been investigated in the context of the present theoretical framework in Refs. [18,25]. A hydrogen maser was used as a reference in the clock-comparison experiment of Prestage et al. The conventional $\mathrm{H}$-maser line involves atomic states with $m_{F}=0$ and hence is insensitive to the parameters for Lorentz violation, which simplifies the resulting experimental bounds. Other ground-state hyperfine lines in hydrogen involve states with $m_{F}= \pm 1$, and they depend on parameters for Lorentz violation according to Eq. (5) of Ref. [25]. At leading order, the sidereal variations of these hyperfine lines are governed by the strength and orientation of the applied magnetic field and the combinations $\widetilde{b}_{J}^{e} \pm \widetilde{b}_{J}^{p}$. Experiments searching for this dependence, perhaps with trapped hydrogen or a hydrogen maser, have the potential to yield calculable bounds. Moreover, since no neutrons are involved, only electron and proton parameters for Lorentz violation would appear.

Among other atoms and ions involving relatively few particles is deuterium. To our knowledge, no clock-comparison experiments have been performed with deuterium. However, an experiment by Wineland and Ramsey [42] studied transition frequencies in a deuterium maser. The transition $\left|F, m_{F}\right\rangle \equiv\left|\frac{3}{2}, \frac{1}{2}\right\rangle \rightarrow\left|\frac{1}{2},-\frac{1}{2}\right\rangle$ was compared when the (weak) applied magnetic field was parallel and antiparallel to the Earth's gravitational field. The result constrains the possible frequency difference to about $200 \mu \mathrm{Hz}$. This experiment was performed to bound the gravitational dipole moment of the deuteron, for which the orientation of the magnetic field relative to a nonrotating frame is irrelevant, and a useful bound on Lorentz violation is difficult to extract from it. However, the possibility of using a deuterium maser as one or both clocks in an experiment to bound Lorentz violation is worth consideration because the neutral deuterium atom is relatively well understood from a theoretical viewpoint. It consists of a deuteron $(I=1)$ and an electron $\left(l_{j}=s_{1 / 2}\right)$, which can combine into states with $F=\frac{3}{2}$ or $F=\frac{1}{2}$ [43]. Explicit forms of the deuteron wave function exist [44] and could in principle be used to calculate the coefficients in Eq. (10) for each particle species. The deuterium atom therefore provides another example of a substance that could produce a calculable bound. However, the deuterium energy-level shifts are sensitive to parameters for Lorentz violation involving all particle species, so any bound attained is unlikely to be clean in the sense defined above.

Perhaps the ideally clean clock-comparison experiment would be one for which one atomic or ionic species is insensitive to Lorentz violation and the other is sensitive to only one of the minimal set of parameters for Lorentz violation $\widetilde{b}_{J}^{w}, \widetilde{c}_{Q, J}^{w}, \widetilde{c}_{-}^{w}, \widetilde{c}_{X Y}^{w}, \widetilde{d}_{J}^{w}, \widetilde{g}_{D, J}^{w}, \widetilde{g}_{Q, J}^{w}, \widetilde{g}_{-}^{w}, \widetilde{g}_{X Y}^{w}$ discussed in the analysis of Sec. II. In practice, however, this ideal is unlikely to be attainable. Insensitive systems such as the hydrogen maser do exist, and in principle an insensitive system could be obtained for any substance by aligning the applied magnetic field with the Earth's rotation axis. However, sensitivity to only one of the minimal parameters for Lorentz violation is difficult to achieve. For example, if a nonzero effect on the energy levels of an atom or ion involves $\widetilde{b}_{J}^{w}$ then it also involves $\widetilde{d}_{J}^{w}$. Comparisons of bounds from different experiments may permit the extraction of a bound on a single parameter for Lorentz violation, but the issue of the calculability of the coefficients would again play an important role.

An interesting option for improving both the calculability and the cleanliness of bounds is to consider atoms or (positive or negative) ions for which there is reason to believe that the energy shifts depend solely or largely on a single valence particle $w$. The presence of only one relevant particle can simplify calculations, and substances of this type would also be relatively clean because only those parameters for Lorentz violation for a particular species $w$ would be involved in the bound.

It is relatively straightforward to identify atoms or ions for which the special species $w$ is an electron, since it suffices to use substances of nuclear spin zero (or nuclear spin $\frac{1}{2}$, if only bounds on quadrupole energy shifts for the electron are considered). These bounds would be of definite interest, even if the precision attainable were less than in experiments with hyperfine transitions. 
TABLE III. Substances with sensitivity to parameters for Lorentz violation for a single particle species.

\begin{tabular}{|c|c|c|c|c|c|c|c|c|c|c|c|c|c|c|c|c|c|c|c|}
\hline & & & & oton & nsiti & $y$ on & & & & & & & & on se & tivity & & & & \\
\hline & $A$ & $Z$ & $N$ & $I$ & $D_{p}$ & $Q_{p}$ & $D_{n}$ & $Q_{n}$ & & & $A$ & $Z$ & $N$ & $I$ & $D_{p}$ & $Q_{p}$ & $D_{n}$ & $Q_{n}$ & \\
\hline $\mathrm{H}$ & 1 & 1 & 0 & $1 / 2$ & $\mathrm{Y}$ & - & - & - & $\star \star$ & $n$ & 1 & 0 & 1 & $1 / 2$ & - & - & $\mathrm{Y}$ & - & $\star \star$ \\
\hline $\mathrm{N}$ & 15 & 7 & 8 & $1 / 2$ & $\mathrm{Y}$ & - & - & - & $\star$ & $\mathrm{He}$ & 3 & 2 & 1 & $1 / 2$ & - & - & $\mathrm{Y}$ & - & $\star \star$ \\
\hline $\mathrm{P}$ & 31 & 15 & 16 & $1 / 2$ & $\mathrm{Y}$ & - & - & - & & $\mathrm{C}$ & 13 & 6 & 7 & $1 / 2$ & - & - & $\mathrm{Y}$ & - & \\
\hline $\mathrm{Y}$ & 89 & 39 & 50 & $1 / 2$ & $\mathrm{Y}$ & - & - & - & $\star$ & $\mathrm{Si}$ & 29 & 14 & 15 & $1 / 2$ & - & - & $\mathrm{Y}$ & - & \\
\hline $\mathrm{Rh}$ & 103 & 45 & 58 & $1 / 2$ & $\mathrm{Y}$ & - & - & - & & $\mathrm{Sn}$ & 115 & 50 & 65 & $1 / 2$ & - & - & $\mathrm{Y}$ & - & $\star$ \\
\hline $\mathrm{Tm}$ & 169 & 69 & 100 & $1 / 2$ & $\mathrm{Y}$ & - & - & - & & $\mathrm{Sn}$ & 117 & 50 & 67 & $1 / 2$ & - & - & $\mathrm{Y}$ & - & $\star$ \\
\hline B & 11 & 5 & 6 & $3 / 2$ & $\mathrm{Y}$ & $\mathrm{Y}$ & - & - & & $\mathrm{Sn}$ & 119 & 50 & 69 & $1 / 2$ & - & - & $\mathrm{Y}$ & - & $\star$ \\
\hline $\mathrm{Al}$ & 27 & 13 & 14 & $5 / 2$ & $\mathrm{Y}$ & $\mathrm{Y}$ & - & - & & $\mathrm{Yb}$ & 171 & 70 & 101 & $1 / 2$ & - & - & $\mathrm{Y}$ & - & \\
\hline $\mathrm{Cl}$ & 37 & 17 & 20 & $3 / 2$ & $\mathrm{Y}$ & $\mathrm{Y}$ & - & - & $\star$ & $\mathrm{Pb}$ & 207 & 82 & 125 & $1 / 2$ & - & - & $\mathrm{Y}$ & - & $\star$ \\
\hline $\mathrm{K}$ & 39 & 19 & 20 & $3 / 2$ & $\mathrm{Y}$ & $\mathrm{Y}$ & - & - & $\star$ & $\mathrm{O}$ & 17 & 8 & 9 & $5 / 2$ & - & - & $\mathrm{Y}$ & $\mathrm{Y}$ & $\star \star$ \\
\hline V & 51 & 23 & 28 & $7 / 2$ & $\mathrm{Y}$ & $\mathrm{Y}$ & - & - & $\star$ & $\mathrm{S}$ & 33 & 16 & 17 & $3 / 2$ & - & - & $\mathrm{Y}$ & $\mathrm{Y}$ & \\
\hline Co & 59 & 27 & 32 & $7 / 2$ & $\mathrm{Y}$ & $\mathrm{Y}$ & - & - & & $\mathrm{Ca}$ & 41 & 20 & 21 & $7 / 2$ & - & - & $\mathrm{Y}$ & $\mathrm{Y}$ & $\star \star$ \\
\hline $\mathrm{Ga}$ & 69 & 31 & 38 & $3 / 2$ & $\mathrm{Y}$ & $\mathrm{Y}$ & - & - & & $\mathrm{Ca}$ & 43 & 20 & 23 & $7 / 2$ & - & - & $\mathrm{Y}$ & $\mathrm{Y}$ & $\star$ \\
\hline $\mathrm{Ga}$ & 71 & 31 & 40 & $3 / 2$ & $\mathrm{Y}$ & $\mathrm{Y}$ & - & - & & $\mathrm{Ni}$ & 61 & 28 & 33 & $3 / 2$ & - & - & $\mathrm{Y}$ & $\mathrm{Y}$ & $\star$ \\
\hline $\mathrm{Rb}$ & 87 & 37 & 50 & $3 / 2$ & $\mathrm{Y}$ & $\mathrm{Y}$ & - & - & $\star$ & $\mathrm{Ge}$ & 73 & 32 & 41 & $9 / 2$ & - & - & $\mathrm{Y}$ & $\mathrm{Y}$ & \\
\hline In & 113 & 49 & 64 & $9 / 2$ & $\mathrm{Y}$ & $\mathrm{Y}$ & - & - & & $\mathrm{Sr}$ & 87 & 38 & 49 & $9 / 2$ & - & - & $\mathrm{Y}$ & $\mathrm{Y}$ & \\
\hline $\mathrm{Sb}$ & 121 & 51 & 70 & $5 / 2$ & $\mathrm{Y}$ & $\mathrm{Y}$ & - & - & & $\mathrm{Zr}$ & 91 & 40 & 51 & $5 / 2$ & - & - & $\mathrm{Y}$ & $\mathrm{Y}$ & $\star$ \\
\hline $\mathrm{La}$ & 139 & 57 & 82 & $7 / 2$ & $\mathrm{Y}$ & $\mathrm{Y}$ & - & - & $\star$ & $\mathrm{Gd}$ & 155 & 64 & 91 & $3 / 2$ & - & - & $\mathrm{Y}$ & $\mathrm{Y}$ & \\
\hline $\operatorname{Pr}$ & 141 & 59 & 82 & $5 / 2$ & $\mathrm{Y}$ & $\mathrm{Y}$ & - & - & $\star$ & $\mathrm{Gd}$ & 157 & 64 & 93 & $3 / 2$ & - & - & $\mathrm{Y}$ & $\mathrm{Y}$ & \\
\hline $\mathrm{Re}$ & 185 & 75 & 110 & $5 / 2$ & $\mathrm{Y}$ & $\mathrm{Y}$ & - & - & & $\mathrm{Er}$ & 167 & 68 & 99 & $7 / 2$ & - & - & $\mathrm{Y}$ & $\mathrm{Y}$ & \\
\hline $\mathrm{Re}$ & 187 & 75 & 112 & $5 / 2$ & $\mathrm{Y}$ & $\mathrm{Y}$ & - & - & & $\mathrm{Yb}$ & 173 & 70 & 103 & $5 / 2$ & - & - & $\mathrm{Y}$ & $\mathrm{Y}$ & \\
\hline $\mathrm{Bi}$ & 209 & 83 & 126 & $9 / 2$ & $\mathrm{Y}$ & $\mathrm{Y}$ & - & - & $\star \star$ & $\mathrm{U}$ & 235 & 92 & 143 & $7 / 2$ & - & - & $\mathrm{Y}$ & $\mathrm{Y}$ & \\
\hline
\end{tabular}

For the case where the special species $w$ is a nucleon, one can generate a list of nuclei for which one might theoretically expect relatively calculable and clean bounds. We consider here substances for which dipole and quadrupole energy shifts depend only on one nucleon species and where there is reason to believe that only one valence nucleon determines the nuclear sensitivity to Lorentz violation. Table III provides a list of naturally abundant isotopes satisfying these criteria. The table has been prepared using only a relatively small set of assumptions about nuclear properties: spin- $\frac{1}{2}$ nuclei are assumed to be insensitive to quadrupole effects, while those with a closed shell for a given nucleon species are assumed to be insensitive to parameters for Lorentz violation for that species. Note in particular that the Schmidt model has not been used. To avoid complicating the bounds through sensitivity to electron parameters for Lorentz violation, experiments on any of the substances listed would need to involve suitable electronic configurations without Lorentz-violating contributions to the relevant transition frequencies. The reader is warned that the table makes no allowance for possible experimental difficulties involved in using these substances.

Substances sensitive to proton parameters for Lorentz violation are listed on the left-hand side of Table III, while those sensitive to neutron parameters are listed on the right-hand side. The quantities $A, Z, N$, and $I$ designate atomic weight, proton number, neutron number, and nuclear spin, respectively. A symbol $Y$ in a column labeled $D_{w}$ or $Q_{w}$ indicates sensitivity of the dipole or quadrupole energy shifts of the substance, respectively, to parameters for Lorentz violation for particles of type $w$. In the left (right) half of this table, all appearances of $Y$ correspond to an odd proton (neutron) number, and the neutron (proton) number is closed-shell [45]. Substances designated by a star have magic neutron (proton) number, while substances designated by two stars have both magic neutron (proton) number and proton (neutron) number equal to a magic number plus one. It seems plausible that these substances are most likely to have nuclear sensitivity to Lorentz violation depending only on a single valence proton (neutron). The case of ${ }^{91} \mathrm{Zr}$ is an exception, in that the neutron number is a magic number plus one, but the proton number is not magic. Although it is not a naturally abundant substance, we have included ${ }^{41} \mathrm{Ca}$ in the table because it is relatively stable (lifetime $\simeq 10^{5} \mathrm{yr}$ ) and it has magic proton number and neutron number equal to a magic number plus one. We have also included the neutron itself in the table for completeness, although technical challenges would need to be overcome to perform Lorentzviolation experiments with (cold) neutrons.

For future clock-comparison experiments, the dual nuclear Zeeman ${ }^{3} \mathrm{He}^{129} \mathrm{Xe}$ maser already in operation [46] could provide an interesting limit on neutron parameters for Lorentz violation because the $I=\frac{1}{2}$ nucleus ${ }^{129} \mathrm{Xe}$ is sensitive to dipole energy shifts from neutron parameters (in a complete nuclear model, it would probably also be sensitive to dipole energy shifts from proton parameters). Within the Schmidt model, the coefficients $\beta_{n}, \gamma_{n}, \delta_{n}, \kappa_{n}, \lambda_{n}$ for both ${ }^{3} \mathrm{He}$ and ${ }^{129} \mathrm{Xe}$ are identical, which would lead to a relatively clean bound. Suppose an experiment with the quantization 
axis in the equatorial plane produces a bound of $\varepsilon_{1, J}, J$ $=X, Y$, on sidereal variations of the free-running ${ }^{3} \mathrm{He}$ frequency using ${ }^{129} \mathrm{Xe}$ as a reference. Within the Schmidt model, we find this would yield the bounds

$$
\left|-3.5 \widetilde{b}_{J}^{n}+0.012 \widetilde{d}_{J}^{n}+0.012 \widetilde{g}_{D, J}^{n}\right| \lesssim 2 \pi \varepsilon_{1, J},
$$

where the ratio of gyromagnetic ratios has been taken as $g^{3} / g^{129} \simeq 2.75$. The factor of -3.5 is relatively large and compares favorably with the corresponding factor of $-\frac{2}{3}$ for the ${ }^{199} \mathrm{Hg}_{-}{ }^{133} \mathrm{Cs}$ case, so even a comparable precision for $\varepsilon_{1, J}$ using the dual ${ }^{3} \mathrm{He}^{-129} \mathrm{Xe}$ maser would represent an improved constraint on parameters for Lorentz violation by more than a factor of 5 .

Another interesting possibility would emerge from the development of a dual ${ }^{3} \mathrm{He}-{ }^{21} \mathrm{Ne}$ maser or a dual ${ }^{3} \mathrm{He}-{ }^{87} \mathrm{Rb}$ maser [47]. Table III shows that ${ }^{3} \mathrm{He}$ is sensitive purely to dipole energy shifts from neutron parameters for Lorentz violation. The ${ }^{3} \mathrm{He}$ and quadrupole ${ }^{21} \mathrm{Ne}$ sensitivities are discussed above and in the Appendix in the context of the experiment of Chupp et al. The dipole ${ }^{21} \mathrm{Ne}$ sensitivity within the Schmidt model includes $\widetilde{b}_{J}^{n}, \widetilde{d}_{J}^{n}, \widetilde{g}_{D, J}^{n}$, and $\widetilde{c}_{Q, J}^{n}$, though in a realistic nuclear model ${ }^{21} \mathrm{Ne}$ would probably also be sensitive to parameters for Lorentz violation for the proton. Table III also shows that ${ }^{87} \mathrm{Rb}$ is a theoretically favorable substance. A quadrupole measurement in a ${ }^{3} \mathrm{He}-{ }^{87} \mathrm{Rb}$ maser using the $\left|\frac{3}{2}, \frac{3}{2}\right\rangle \rightarrow\left|\frac{3}{2}, \frac{1}{2}\right\rangle$ or $\left|\frac{3}{2},-\frac{1}{2}\right\rangle \rightarrow\left|\frac{3}{2},-\frac{3}{2}\right\rangle{ }^{87} \mathrm{Rb}$ transitions (but not both with equal weight) therefore has the potential to provide an unusually clean bound on proton parameters for Lorentz violation.

\section{SUMMARY}

In this work, we have analyzed clock-comparison experiments in the context of a general extension of the standard model and quantum electrodynamics allowing for Lorentz and CPT violation. In this theory, both dipole and quadrupole shifts of atomic or ionic energy levels are predicted and would produce sidereal and semisidereal time dependences of the signal. We have obtained explicit formulas for these effects that can be applied to existing and future experiments and have demonstrated that the experimental results already available place interesting constraints on certain combinations of the parameters in the theory.

Our expressions show that experiments performed with different atoms or ions typically test inequivalent quantities as a result of possible variations of the parameters for Lorentz violation with the species of elementary particle. Indeed, no two of the experimental bounds obtained to date involve identical linear combinations of parameters, and the sensitivities of the two most recent experiments have no overlap at all.

The variety of high-precision experiments already performed allows a region of the parameter space to be excluded. However, the exact specification of this region is theoretically uncertain because for the most part of the bounds are obtained from atoms or ions with relatively involved nuclear structure. Some regions of the attainable pa- rameter space are as yet unconstrained by clock-comparison experiments.

We have considered the issues involved in producing theoretically favorable bounds, and have listed some naturally abundant substances that may be of potential interest for future tests. The exceptional degree of precision attainable offers potential sensitivity to Lorentz-violating effects from the Planck scale and ensures that future clockcomparison experiments remain among the most attractive possibilities for detection of any nonzero effect that might exist in nature.

\section{ACKNOWLEDGMENTS}

We thank R. Bluhm, L. R. Hunter, J. D. Prestage, R. E. Stoner, R. Walsworth, and D. J. Wineland for useful discussions. This work was supported in part by the United States Department of Energy under Grant No. DE-FG0291ER40661.

\section{APPENDIX: SPECIFIC EXPERIMENTS}

This appendix contains remarks specific to the experiments discussed in Sec. III. Some issues relevant to the calculations leading to Eq. (21) and Table I are presented. Each experiment is considered under a separate heading.

All the experiments we consider apply a constant magnetic field of magnitude $B$ that fixes the quantization axis of the atom or ion $W$. Following the discussion in Sec. II D, we define the $z$ axis to be aligned with this field. Let $\vec{I}, \vec{J}$, and $\vec{F}$ represent the nuclear, electronic, and total angular momentum of $W$, respectively. Where relevant, we denote the corresponding quantum numbers by $I, J$, and $F$. The degree to which $W$ is in an eigenstate of these operators is governed by a parameter $\zeta \approx\left(g_{J}-g_{I}\right) \mu_{B} B / E_{\mathrm{hfs}}$, where $g_{J}$ is the Landé $g$ factor of the electron cloud, $g_{I}$ is the Landé $g$ factor of the nucleus, $\mu_{B}$ is the Bohr magneton, and $E_{\mathrm{hfs}}$ is the hyperfine splitting of the atom [48]. For $J \neq 0,\left|g_{I}\right| \ll\left|g_{J}\right|$. In most experiments that we consider, the applied magnetic field is small compared to the internal interactions of $W$. In this case, $|\zeta| \ll 1, W$ is approximately in an eigenstate of $\vec{F}^{2}$ and $F_{z}$ with quantum numbers $F$ and $m_{F}$, and the error introduced by approximating wave functions as eigenfunctions of $\vec{F}^{2}$ is suppressed by $\zeta^{2} \sim 10^{-12}$. However, in the experiment of Prestage et al. a relatively large magnetic field is applied to the ${ }^{9} \mathrm{Be}^{+}$ion. In this case, $|\zeta| \gg 1$, the ion is approximately in an eigenstate of $I_{z}$ and $J_{z}$ with quantum numbers $m_{I}$ and $m_{J}$, and the error due to approximating the ${ }^{9} \mathrm{Be}^{+}$wave function to be an eigenfunction of $J_{z}$ and $I_{z}$ is suppressed by $1 / \zeta^{2}$ $\sim 10^{-5}$.

\section{1. ${ }^{9} \mathrm{Be}^{+}$and $H$ maser}

The experiment of Prestage et al. [3] measures the frequency $\nu$ of a ${ }^{9} \mathrm{Be}^{+}$transition in a large $(0.8194 \mathrm{~T})$ magnetic field relative to the frequency of a hydrogen maser. The ${ }^{9} \mathrm{Be}^{+}$transition is $\left|m_{I}, m_{J}\right\rangle=\left|-\frac{3}{2},+\frac{1}{2}\right\rangle \rightarrow\left|-\frac{1}{2},+\frac{1}{2}\right\rangle$. The $H$-maser transition is $\left|F, m_{F}\right\rangle=|1,0\rangle \rightarrow|0,0\rangle$. The experiment searches for a time variation in the frequency $\nu$ of the form 
$\nu=\nu_{0}+A_{k} P_{k}[\cos \beta(t)]$, where $A_{1}, A_{2}$, and $A_{3}$ are constants, $P_{k}$ denotes the $k$ th Legendre polynomial, and $\beta(t)$ is the angle between the quantization axis and a direction of spatial anisotropy. The limits obtained on the three quantities $\left|A_{k}\right|$ are approximately $100 \mu \mathrm{Hz}$.

Within the theoretical framework of the standard-model extension, the standard hydrogen-maser frequency is unaffected by Lorentz violation [25]. The sensitivity to Lorentz violations therefore resides entirely in the ${ }^{9} \mathrm{Be}^{+}$ion. This ion has a nucleus with $I=\frac{3}{2}$ surrounded by an electron cloud with $J=\frac{1}{2}$, so the nucleus could be sensitive in principle to dipole, quadrupole, and octupole energy shifts, while the electron cloud could be sensitive to dipole energy shifts. However, the transition frequency in the experiment is effectively insensitive to electron parameters for Lorentz violation because $\Delta m_{J} \simeq 0$. The formulas of Sec. II D therefore apply with $F$ replaced by the nuclear spin $I$.

The theoretical time variation $\nu(t)$ of the frequency $\nu$ can be obtained by applying Eq. (19) to the two energy levels involved. In the experiment, the magnetic field is at an angle of $\chi \simeq 118^{\circ}$ with respect to the Earth's rotation axis. The various constants defined in Eq. (10) can be calculated approximately with the methods of Sec. IIC. The ${ }^{9} \mathrm{Be}^{+}$nucleus consists of four protons and five neutrons. The Schmidt model predicts that a single neutron in a $p_{3 / 2}$ state carries the entire nuclear angular momentum, in agreement with the shell-model prediction that each valence nucleon is in a $p_{3 / 2}$ state. The resulting values of the constants are given in Table I.

The theoretical expression for $\nu(t)$ can be compared to the experimental fit for $\nu$. This gives bounds of the form in Eq. (21), where the constants are specified in Table I.

\section{2. ${ }^{201} \mathrm{Hg}$ and ${ }^{199} \mathrm{Hg}$}

The experiment of Lamoreaux et al. [4] compares precession frequencies of ${ }^{201} \mathrm{Hg}$ and ${ }^{199} \mathrm{Hg}$ atoms in a weak magnetic field. The electron clouds of both types of atom have $J=0$ in the ground state, so the corresponding atomic states can be labeled $\left|I, m_{I}\right\rangle$. The precession frequencies arise from $\Delta m_{I}=1$ transitions. The experiment searches for possible sidereal or semisidereal time variations in the frequency difference, yielding an upper bound of about half a microhertz.

The ${ }^{199} \mathrm{Hg}$ nucleus has $I=\frac{1}{2}$ and is sensitive only to dipole shifts, while the ${ }^{201} \mathrm{Hg}$ nucleus has $I=\frac{3}{2}$ and is sensitive to dipole, quadrupole, and octupole shifts. The formulas of Sec. II D apply with $F$ replaced by the nuclear spin $I$. The possible time variations in the observed frequency difference can be found within the present framework by using Eq. (19) for each of the energy levels involved in the transitions. The magnetic field in the experiment lies in the Earth's equatorial plane, so $\chi=\pi / 2$ and many of the geometrical factors described in Sec. II D simplify.

The ${ }^{201} \mathrm{Hg}$ nucleus has 80 protons and 121 neutrons, while the ${ }^{199} \mathrm{Hg}$ nucleus has 80 protons and 119 neutrons. The nuclear shell model predicts that the ground-state properties of ${ }^{201} \mathrm{Hg}$ and ${ }^{199} \mathrm{Hg}$ are determined by the neutrons. This implies the vanishing of all coefficients of the form (10) for the proton and would mean that both isotopes are sensitive only to neutron parameters for Lorentz violation. Both isotopes have valence protons and neutrons, however, so it is likely that a more realistic model would produce nonzero coefficients (10) for protons too and therefore that both nuclei are sensitive to proton and neutron parameters for Lorentz violation.

The Schmidt model indicates that the angular momentum of the ${ }^{199} \mathrm{Hg}$ nucleus is carried by a single neutron in a $p_{1 / 2}$ state. Naively, this is at odds with the shell model, which implies each valence neutron is in an $i_{13 / 2}$ state. However, when there are nearly degenerate states with different orbital angular momenta $l$, the shell model also suggests that protons or neutrons prefer to pair in states of high $l$. This would mean that the $i_{13 / 2}$ shell is closed preferentially to shells immediately below it with lower $l$. The shells immediately below $i_{13 / 2}$ are $p_{1 / 2}$ and $p_{3 / 2}$, so the Schmidt-model prediction is compatible with that from the shell model. A similar discussion applies to the ${ }^{201} \mathrm{Hg}$ nucleus, except that the single neutron is in a $p_{3 / 2}$ state.

Calculating the coefficients in Eq. (10) according to the methods of Sec. IIC yields the results given in Table I. In converting the actual experimental bounds to the form of Eq. (21) with the constants given in Table I, we have for simplicity approximated the ${ }^{201} \mathrm{Hg}$ precession frequency as involving only the transition $\left|\frac{3}{2}, \frac{3}{2}\right\rangle \rightarrow\left|\frac{3}{2}, \frac{1}{2}\right\rangle$. A more accurate expression involving also the transition $\left|\frac{3}{2},-\frac{1}{2}\right\rangle \rightarrow\left|\frac{3}{2},-\frac{3}{2}\right\rangle$ could be obtained following the detailed analysis in Ref. [4], but the results remain essentially unchanged. Note that the nonzero value of $\mathrm{v}$ in Table I reflects the ratio of gyromagnetic ratios of the two $\mathrm{Hg}$ isotopes, $g_{201} / g_{199} \simeq-0.37$, and the corresponding dependence of the sidereal bounds on both ${ }^{201} \mathrm{Hg}$ and ${ }^{199} \mathrm{Hg}$. In contrast, the semisidereal bounds depend only on ${ }^{201} \mathrm{Hg}$, in accordance with its sensitivity to quadrupole shifts.

\section{3. ${ }^{21} \mathrm{Ne}$ and ${ }^{3} \mathrm{He}$}

The experiment of Chupp et al. [5] searches for quadrupole shifts in ${ }^{21} \mathrm{Ne}$ precession frequencies relative to a reference precession frequency in ${ }^{3} \mathrm{He}$, placing a bound of about half a microhertz on possible semisidereal variations. The electron clouds of ${ }^{21} \mathrm{Ne}$ and ${ }^{3} \mathrm{He}$ both have $J=0$ in the ground state, so in a weak magnetic field only the nuclear angular momenta are relevant and the corresponding atomic state can be labeled $\left|I, m_{I}\right\rangle$. The experiment is insensitive to electron parameters for Lorentz violation, and the formulas of Sec. II D apply with $F$ replaced by the nuclear spin $I$. The ${ }^{3} \mathrm{He}$ nucleus has $I=\frac{1}{2}$ and is therefore sensitive only to dipole shifts, while the ${ }^{21} \mathrm{Ne}$ nucleus has $I=\frac{3}{2}$ with sensitivity in principle to dipole, quadrupole, and octupole shifts.

The shift in each energy level is given by Eq. (19) and can be used to deduce the possible time variations of the signal frequency in the present theoretical framework. The magnetic field in the experiment is perpendicular to the Earth's rotation axis, so $\chi=\pi / 2$ and many geometrical factors in Sec. II D vanish. Since the experiment bounds only semisidereal frequencies, which are independent of dipole energy shifts, the possible dipole energy shifts in both ${ }^{21} \mathrm{Ne}$ and ${ }^{3} \mathrm{He}$ 
have no effect on the experiment.

The ${ }^{21} \mathrm{Ne}$ nucleus consists of 10 protons and 11 neutrons. According to the shell model, the ground-state properties of ${ }^{21} \mathrm{Ne}$ depend only on the neutrons, which suggests all coefficients of the type (10) for the proton must vanish and would imply the experiment is insensitive to proton parameters for Lorentz violation. However, neither the protons nor the neutrons lie in a closed nuclear shell, so it is likely that in reality the experiment does have sensitivity to proton parameters for Lorentz violation.

In the Schmidt model, the ground-state properties of ${ }^{21} \mathrm{Ne}$ and ${ }^{3} \mathrm{He}$ are determined by a single neutron in a $p_{3 / 2}$ and an $s_{1 / 2}$ state, respectively. This assignment for ${ }^{21} \mathrm{Ne}$ would appear to contradict the shell-model prediction that each valence neutron is in a $d_{5 / 2}$ state. It is, however, plausible within the shell model that the $d_{5 / 2}$ shell is closed preferentially to the states immediately below it in energy, namely, $p_{1 / 2}$ and $p_{3 / 2}$. This argument for ${ }^{21} \mathrm{Ne}$ is weaker than the corresponding argument for ${ }^{199} \mathrm{Hg}$ in Sec. 2 of this appendix because the $p_{1 / 2}$ shell relevant for ${ }^{21} \mathrm{Ne}$ is not merely closed but corresponds also to a magic number. Since in any event a complete shell-model calculation would still be inadequate in that the dependence on proton parameters for Lorentz violation would be missing, we present only the Schmidt-model values in this work.

The results of the calculation produce bounds of the form of the last two equations in Eq. (21), with coefficients given in Table I. To match the actual experimental bounds to this form, we have for simplicity approximated the ${ }^{21} \mathrm{Ne}$ precession frequency as involving only the transition $\left|\frac{3}{2}, \frac{3}{2}\right\rangle$ $\rightarrow\left|\frac{3}{2}, \frac{1}{2}\right\rangle$. A more accurate expression involving also the transition $\left|\frac{3}{2},-\frac{1}{2}\right\rangle \rightarrow\left|\frac{3}{2},-\frac{3}{2}\right\rangle$ could be obtained with the methods of Ref. [5], but this has no substantial effect on the results.

\section{4. ${ }^{199} \mathrm{Hg}$ and ${ }^{133} \mathrm{Cs}$}

The experiment of Berglund et al. [6] bounds the possible sidereal time dependence of ${ }^{199} \mathrm{Hg}$ and ${ }^{133} \mathrm{Cs}$ precession frequencies. The procedure uses a weak magnetic field to split the ground states of the ${ }^{199} \mathrm{Hg}$ and ${ }^{133} \mathrm{Cs}$ atoms. Denote the associated frequencies by $\nu^{133}$ and $\nu^{199}$. The experiment measures the difference $\Delta B$ between the effective magnetic fields measured by the ${ }^{199} \mathrm{Hg}$ and ${ }^{133} \mathrm{Cs}$ atoms. This can be written $\Delta B \equiv \nu^{199} / g^{199}-\nu^{133} / g^{133}$, where $g^{199}$ $\simeq 0.759 \mathrm{kHz} / \mathrm{G}$ and $g^{133} \simeq 350 \mathrm{kHz} / \mathrm{G}$ are the gyromagnetic ratios of ${ }^{199} \mathrm{Hg}$ and ${ }^{133} \mathrm{Cs}$, respectively. We take the experimental bound obtained as a limit on possible sidereal variations of the frequency difference $g^{199} \Delta B$ at the level of about $100 \mathrm{nHz}$.

The electron cloud of the ${ }^{199} \mathrm{Hg}$ atom in its ground state has $J=0$ and its nucleus has $I=\frac{1}{2}$, so it is sensitive only to dipole shifts and is insensitive to electron parameters for Lorentz violation. See Sec. 2 of this appendix for more information about ${ }^{199} \mathrm{Hg}$. In contrast, the ground state of the ${ }^{133} \mathrm{Cs}$ atom has an electron cloud with 55 electrons in a $J$ $=\frac{1}{2}$ state and a nucleus with $I=\frac{7}{2}$. The ${ }^{133} \mathrm{Cs}$ states relevant to the experiment have total angular momentum $F=4$, so in principle sensitivity to nonzero energy shifts of multipolarity up to order 8 would be possible. However, in the present framework the sidereal frequency dependences bounded by the experiment can depend only on dipole and quadrupole energy-level shifts. The relevant shifts leading to possible time variations in the signal are given by Eq. (19). In the experiment, the quantization axis is always perpendicular to the Earth's rotation axis so $\chi=\pi / 2$, which simplifies the formulas in Sec. II D.

The outer electronic shell of ${ }^{133} \mathrm{Cs}$ consists of a single valence electron in a $6 s$ state. Since the closed shells do not contribute to dipole or quadrupole energy shifts, only the valence electron is relevant. It is straightforward to calculate the contributions to the coefficients in Eq. (10) for the electron, using the expressions given in Sec. II C.

The ${ }^{133} \mathrm{Cs}$ nucleus contains 55 protons and 78 neutrons. The shell model suggests that the ${ }^{133} \mathrm{Cs}$ ground-state properties are independent of the neutrons. Since the ${ }^{199} \mathrm{Hg}$ properties do depend on neutrons, even in the shell-model approximation the experimental results are sensitive to contributions from all three species of particle. Moreover, since neither the protons nor the neutrons lie in a closed nuclear shell, the ${ }^{133} \mathrm{Cs}$ atom alone is likely to be sensitive to parameters for Lorentz violation from all three species. For simplicity and definiteness, we limit the analysis in this paper to the Schmidt model, for which the only significant nucleon is a proton in a $g_{7 / 2}$ state (in agreement with the shell model).

In the context of the present framework, the bounds obtained in the experiment take the form of the first two equations in Eq. (21). The values of the coefficients are given in Table I, where the transitions have been taken as $\left|I, m_{I}\right\rangle$ $=\left|\frac{1}{2},+\frac{1}{2}\right\rangle \rightarrow\left|\frac{1}{2},-\frac{1}{2}\right\rangle$ in ${ }^{199} \mathrm{Hg}$ and $\left|F, m_{F}\right\rangle=|4,4\rangle \rightarrow|4,3\rangle$ in

${ }^{133} \mathrm{Cs}$. Note that the parameter $\mathrm{V}$ is small, primarily because the ratio of gyromagnetic ratios $\left|g^{199} / g^{133}\right|$ is small.
[1] V. W. Hughes, H. G. Robinson, and V. Beltran-Lopez, Phys. Rev. Lett. 4, 342 (1960).

[2] R. W. P. Drever, Philos. Mag. 6, 683 (1961).

[3] J. D. Prestage et al., Phys. Rev. Lett. 54, 2387 (1985).

[4] S. K. Lamoreaux et al., Phys. Rev. Lett. 57, 3125 (1986); Phys. Rev. A 39, 1082 (1989).

[5] T. E. Chupp et al., Phys. Rev. Lett. 63, 1541 (1989).

[6] C. J. Berglund et al., Phys. Rev. Lett. 75, 1879 (1995).

[7] The term "clock-comparison experiment"' is used throughout this work to denote a generic class of experiments comparing transition frequencies in atoms or ions, whether the experiment uses actual atomic clocks, sensitive magnetometers, or other techniques.

[8] D. Colladay and V. A. Kostelecký, Phys. Rev. D 55, 6760 (1997); 58, 116002 (1998).

[9] V. A. Kostelecký and S. Samuel, Phys. Rev. Lett. 63, 224 (1989); 66, 1811 (1991); Phys. Rev. D 39, 683 (1989); 40, 1886 (1989); V. A. Kostelecký and R. Potting, Nucl. Phys. B359, 545 (1991); Phys. Lett. B 381, 89 (1996).

[10] See, for example, Particle Data Group, C. Case et al., Eur. 
Phys. J. C 3, 1 (1998)

[11] Some recent developments for several of the approaches to Lorentz and CPT violation are discussed in CPT and Lorentz. Symmetry, edited by V. A. Kostelecký (World Scientific, Singapore, 1999).

[12] Many unconventional theories of gravity (without standardmodel physics), including some containing various possible sources of local Lorentz violation, are reviewed in C. M. Will, Theory and Experiment in Gravitational Physics (Cambridge University Press, Cambridge, 1993).

[13] V. A. Kostelecký and R. Potting, in Gamma Ray-Neutrino Cosmology and Planck Scale Physics, edited by D. B. Cline (World Scientific, Singapore, 1993), hep-th/9211116; Phys. Rev. D 51, 3923 (1995); D. Colladay and V. A. Kostelecký, Phys. Lett. B 344, 259 (1995); Phys. Rev. D 52, 6224 (1995); V. A. Kostelecký and R. Van Kooten, ibid. 54, 5585 (1996).

[14] B. Schwingenheuer et al., Phys. Rev. Lett. 74, 4376 (1995); L. K. Gibbons et al., Phys. Rev. D 55, 6625 (1997); R. Carosi et al., Phys. Lett. B 237, 303 (1990).

[15] OPAL Collaboration, R. Ackerstaff et al., Z. Phys. C 76, 401 (1997); DELPHI Collaboration, M. Feindt et al., Report No. DELPHI 97-98 CONF 80, 1997.

[16] V. A. Kostelecký, Phys. Rev. Lett. 80, 1818 (1998); Phys. Rev. D (to be published), hep-ph/9909554.

[17] P. B. Schwinberg, R. S. Van Dyck, Jr., and H. G. Dehmelt, Phys. Lett. 81A, 119 (1981); R. S. Van Dyck, Jr., P. B. Schwinberg, and H. G. Dehmelt, Phys. Rev. D 34, 722 (1986); L. S. Brown and G. Gabrielse, Rev. Mod. Phys. 58, 233 (1986); R. S. Van Dyck, Jr., P. B. Schwinberg, and H. G. Dehmelt, Phys. Rev. Lett. 59, 26 (1987); G. Gabrielse et al., ibid., 74, 3544 (1995).

[18] R. Bluhm, V. A. Kostelecký, and N. Russell, Phys. Rev. Lett. 79, 1432 (1997); Phys. Rev. D 57, 3932 (1998).

[19] G. Gabrielse et al., in CPT and Lorentz Symmetry, Ref. [11]; Phys. Rev. Lett. 82, 3198 (1999).

[20] H. Dehmelt et al., Phys. Rev. Lett. (to be published), hep-ph/9906262.

[21] R. Mittleman et al., in CPT and Lorentz Symmetry, Ref. [11]; Phys. Rev. Lett. 83, 2116 (1999).

[22] S. M. Carroll, G. B. Field, and R. Jackiw, Phys. Rev. D 41, 1231 (1990).

[23] R. Jackiw and V. A. Kostelecký, Phys. Rev. Lett. 82, 3572 (1999).

[24] M. Charlton et al., Phys. Rep. 241, 65 (1994); Antihydrogen, edited by J. Eades (Baltzer, Geneva, 1993).

[25] R. Bluhm, V. A. Kostelecký, and N. Russell, Phys. Rev. Lett. 82, 2254 (1999).

[26] O. Bertolami et al., Phys. Lett. B 395, 178 (1997).

[27] V. A. Kostelecký and C. D. Lane, J. Math. Phys. (to be published), hep-ph/9909542.

[28] L. L. Foldy and S. A. Wouthuysen, Phys. Rev. 78, 29 (1950).
[29] A. P. Lightman and D. L. Lee, Phys. Rev. D 8, 364 (1973).

[30] H. B. Nielsen and I. Picek, Nucl. Phys. B211, 269 (1983).

[31] S. Coleman and S. Glashow, Phys. Rev. D 59, 116008 (1999).

[32] See, for example, M. E. Rose, Elementary Theory of Angular Momentum (Dover, New York, 1957).

[33] A general discussion of nuclear models can be found in A. Bohr and B. R. Mottelson, Nuclear Structure (Benjamin, New York, 1969).

[34] See, for example, M. Goeppert Mayer and J. H. D. Jensen, Elementary Theory of Nuclear Shell Structure (Wiley, New York, 1955).

[35] Some recent developments in shell models are described in Contemporary Nuclear Shell Models, edited by X.-W. Pan, D. H. Feng, and M. Vallières (Springer-Verlag, New York, 1997).

[36] T. Schmidt, Z. Phys. 106, 358 (1937).

[37] See, for example, J. M. Blatt and V. F. Weisskopf, Theoretical Nuclear Physics (Wiley, New York, 1952).

[38] M. E. Rose and H. A. Bethe, Phys. Rev. 51, 205 (1937).

[39] See, for example, R. M. Green, Spherical Astronomy (Cambridge University Press, Cambridge, England, 1985).

[40] The clock-comparison experiments of Refs. [1-6] are distributed over several decades, so in principle a comparison of their bounds should allow for this precession. We neglect this effect here.

[41] In the absence of Lorentz violation, applying a weak magnetic field $\vec{B}$ to $W$ causes a degenerate energy level to split and a single associated peak to appear in the idealized spectrum, which remains unaffected as the Earth rotates. The Lorentzviolating perturbations can further split this peak and move the location of its centroid, while the Earth's rotation typically causes the centroid to move back and forth and the spacing of the subsidiary peaks to expand and contract. However, in general the link between the multipolarities of the energy shifts and the number of subsidiary peaks or the frequencies of the motion can be involved.

[42] D. J. Wineland and N. F. Ramsey, Phys. Rev. A 5, 821 (1972).

[43] N. F. Ramsey, Molecular Beams (Oxford University Press, New York, 1956).

[44] The deuteron wave function is discussed in, for example, R. Machleidt, K. Holinde, and C. Elster, Phys. Rep. 149, 1 (1987).

[45] In a nuclear shell model, the magic numbers are 2, 8, 20, 28, $50,82,126$, and 184 [34]. A closed-shell number is one corresponding to a closed nuclear shell, in which the protons (neutrons) occupy a state with zero orbital and spin angular momenta: 2, 6, 8, 14, 16, 20, 28,...

[46] R. E. Stoner et al., Phys. Rev. Lett. 77, 3971 (1996).

[47] R. E. Stoner, in CPT and Lorentz Symmetry, Ref. [11]; R. E. Stoner and R. Walsworth, Smithsonian Scholarly Studies proposal, 1998.

[48] G. Breit and I. I. Rabi, Phys. Rev. 38, 2082 (1931). 